\title{
GENERAL MAXIMAL OPERATORS AND THE REVERSE HÖLDER CLASSES
}

\author{
Hiroki Saito and Hitoshi Tanaka \\ Kogakuin University, Academic Support Center \\ 2665-1, Nakanomachi, Hachioji-shi Tokyo, 192-0015, Japan; j1107703@gmail.com \\ National University Corporation Tsukuba University of Technology \\ Research and Support Center on Higher Education for the Hearing and Visually Impaired \\ Kasuga 4-12-7, Tsukuba City, Ibaraki, 305-8521 Japan; htanaka@k.tsukuba-tech.ac.jp
}

\begin{abstract}
By a basis in $\mathbf{R}^{n}$ we mean a collection of open and bounded sets $\mathfrak{B}$. In this paper we show that, if the general maximal operator $M_{\mathfrak{B}}$ is bounded on $L^{p}\left(\mathbf{R}^{n}\right)$ for $p>1$ and the weight $w$ belongs to the reverse Hölder $\mathrm{RH}_{\infty, \mathfrak{B}}$ class, then the weighted maximal operator $M_{\mathfrak{B}, w}$ is bounded on $L^{p}\left(\mathbf{R}^{n}, w\right)$ for $p>1$. When the general basis $\mathfrak{B}$ has dyadic substructure with the Stein property, we investigate the equivalence between the Muckenhoupt class $A_{\infty, \mathfrak{B}}$ and the reverse Hölder class $\mathrm{RH}_{1, \mathfrak{B}}$. We also discuss equivalent ways of defining the reverse Hölder class $\mathrm{RH}_{1, \mathfrak{B}}$.
\end{abstract}

\section{Introduction}

The purpose of this paper is to develop a theory of weights for general maximal operators. We first fix some notations. By weights we will always mean non-negative, locally integrable functions on $\mathbf{R}^{n}$ which are positive on a set of positive measure. Given a measurable set $E$ and a weight $w, w(E)=\int_{E} w(x) d x,|E|$ denotes the Lebesgue measure of $E$ and $\mathbf{1}_{E}$ denotes the characteristic function of $E$. Given $1<p<\infty, p^{\prime}=\frac{p}{p-1}$ will denote the conjugate exponent number of $p$. Let $0<p \leq \infty$ and $w$ be a weight. We define the weighted Lebesgue space $L^{p}\left(\mathbf{R}^{n}, w\right)=L^{p}(w)$ to be a Banach space equipped with the norm (or quasi norm)

$$
\|f\|_{L^{p}(w)}=\left(\int_{\mathbf{R}^{n}}|f(x)|^{p} w(x) d x\right)^{1 / p} .
$$

In their paper [2], Alfonseca, Soria and Vargas proposed that directional maximal operators enjoy an almost-orthogonality principle in $L^{2}\left(\mathbf{R}^{2}\right)$. Motivated by an extension of this interesting property to the setting of radial weights, we considered in [18] the following weights: The weight $w$ is of the form $w(x)=w_{0}(|x|), x \in \mathbf{R}^{2}$, where $w_{0}:[0, \infty) \rightarrow[0, \infty)$ satisfies

$$
\sup _{r_{1}<r<r_{2}} w_{0}(r) \leq \frac{C}{r_{2}-r_{1}} \int_{r_{1}}^{r_{2}} w_{0}(r) d r
$$

for all $0<r_{1}<r_{2}<\infty$. Notice that $r^{a}$ with $a>0$ satisfies this condition. For this weight $w$ we proved the following geometrical fact which was observed in [1] when $w \equiv 1$.

https://doi.org/10.5186/aasfm.2017.4227

2010 Mathematics Subject Classification: Primary 42B25; Secondary 42B35.

Key words: general maximal operator, Gehring lemma, Fujii-Wilson constant, Muckenhoupt weight class; reverse Hölder weight class, Stein property.

The first author is supported by Grant-in-Aid for Young Scientists (B) (15K17551), the Japan Society for the Promotion of Science. The second author is supported by Grant-in-Aid for Scientific Research (C) (15K04918), the Japan Society for the Promotion of Science. 
Let $0<\theta_{1}, \theta_{2}<\pi / 4$. Let

$$
\omega_{0}=(1,0), \quad \omega_{1}=\left(\cos \theta_{1}, \sin \theta_{1}\right) \quad \text { and } \quad \omega_{2}=\left(\cos \left(-\theta_{2}\right), \sin \left(-\theta_{2}\right)\right) .
$$

Let $B$ be a rectangle whose longest side is parallel to $\omega_{1}$ and let $R$ be a rectangle whose longest side is parallel to $\omega_{2}$. Suppose that $B \cap R \neq \emptyset$ and that the long side length of $B$ is bigger than that of $R$. Then there exists a rectangle $\widetilde{R} \supset R$ whose longest side is parallel to $\omega_{0}$ such that

$$
\frac{w(R \cap B)}{w(R)} \leq C \frac{w(\widetilde{R} \cap B)}{w(\widetilde{R})},
$$

where the constant $C$ is independent of $\theta_{1}, \theta_{2}, B$ and $R$.

One of the main ingredients for the proof of this somehow complicated fact is that, $w$ fulfills a quite nice formula:

$$
\frac{w(R)}{|R|} \approx \frac{1}{\operatorname{rad}(R)} \int_{r_{1}(R)}^{r_{2}(R)} w_{0}(r) d r
$$

for any rectangle $R \subset \mathbf{R}^{2}$, where

$$
r_{1}(R)=\inf _{x \in R}|x|, \quad r_{2}(R)=\sup _{x \in R}|x| \quad \text { and } \quad \operatorname{rad}(R)=r_{2}(R)-r_{1}(R) .
$$

By the use of these relations (1.1) and (1.2), in [17], the first author established the logarithmic boundedness of weighted small Kakeya maximal operators on $L^{2}\left(\mathbf{R}^{2}, w\right)$.

Fix $N \gg 1$. For a real number $a>0$ let $\mathfrak{K}_{N, a}$ be the family of all tubes in $\mathbf{R}^{n}, n \geq 2$, which are congruent to the tubes with height $N a$ and width $a$, but with arbitrary directions and centers. Let $\mathfrak{K}_{N}=\bigcup_{a>0} \mathfrak{K}_{N, a}$. For an $f \in L_{\text {loc }}^{1}\left(\mathbf{R}^{n}\right)$ the small Kakeya maximal operator $M_{\mathfrak{K}_{N, a}}$ is defined by

$$
M_{\mathfrak{K}_{N, a}} f(x)=\sup _{T \in \mathfrak{K}_{N, a}} \mathbf{1}_{T}(x) \frac{1}{|T|} \int_{T}|f(y)| d y
$$

and the Kakeya maximal operator $M_{\mathfrak{K}_{N}}$ is defined by

$$
M_{\mathfrak{K}_{N}} f(x)=\sup _{T \in \mathfrak{K}_{N}} \mathbf{1}_{T}(x) \frac{1}{|T|} \int_{T}|f(y)| d y .
$$

It is conjectured that $M_{\mathfrak{K}_{N}}$ is bounded on $L^{n}\left(\mathbf{R}^{n}\right)$ with the operator norm which grows no faster than $\mathrm{O}\left((\log N)^{\alpha_{n}}\right)$ for some $\alpha_{n}>0$ as $N \rightarrow \infty$. In the case $n=2$, this conjecture was solved affirmatively by Córdoba [3] with the exponent $\alpha_{2}=2$ and reproved by Strömberg [16] with $\alpha_{2}=1$. In the higher dimensional case, $n>2$, these estimates were proved so far only for some restricted class of functions; see for example the references in [18].

For a weight $w$ the corresponding weighted maximal operators are defined by

$$
\begin{aligned}
M_{\mathfrak{K}_{N, a}, w} f(x) & =\sup _{T \in \mathfrak{K}_{N, a}} \mathbf{1}_{T}(x) \frac{1}{w(T)} \int_{T}|f(y)| w(y) d y, \\
M_{\mathfrak{K}_{N}, w} f(x) & =\sup _{T \in \mathfrak{K}_{N}} \mathbf{1}_{T}(x) \frac{1}{w(T)} \int_{T}|f(y)| w(y) d y .
\end{aligned}
$$

It is shown in [18] that, if the weight $w$ is of the form $w(x)=|x|^{a}, a>0$, then

$$
\left\|M_{\mathfrak{K}_{N}, w} f\right\|_{L^{2}\left(\mathbf{R}^{2}, w\right)} \leq C \log N\|f\|_{L^{2}\left(\mathbf{R}^{2}, w\right)}
$$

and is also shown in [17] that, if $w$ satisfies (1.1), then for any $a>0$

$$
\left\|M_{\mathfrak{K}_{N, a}, w} f\right\|_{L^{2}\left(\mathbf{R}^{2}, w\right)} \leq C \sqrt{\log N}\|f\|_{L^{2}\left(\mathbf{R}^{2}, w\right)} .
$$


In this paper we shall prove a theorem concerning general maximal operator which implies those two estimates (1.3) and (1.4) as a corollary; see Remark 2.8.

When the case $\mathfrak{B}=\mathfrak{Q}$, the collection of all cubes in $\mathbf{R}^{n}$ with sides parallel to the coordinate axes, it is well known that the Muckenhoupt $A_{p}$ condition characterizes the weighted $L^{p}$ estimate for several important operators; see for example [6]. The reverse Hölder class $\mathrm{RH}_{1, \mathfrak{Q}}$ is closely related to the Muckenhoupt class $A_{\infty, \mathfrak{Q}}$. However, it seems that further much more is known about the Muckenhoupt class $A_{\infty, \mathfrak{Q}}$ than about the reverse Hölder class $\mathrm{RH}_{1, \mathfrak{Q}}$. In [4], Cruz-Uribe and Neugebauer investigated deeply the structure of the reverse Hölder class $\mathrm{RH}_{1, \mathfrak{Q}}$. In this paper we study the structure of the reverse Hölder classes with general basis $\mathfrak{B}$ and prove the equivalence between the Muckenhoupt class $A_{\infty, \mathfrak{B}}$ and the reverse Hölder class $\mathrm{RH}_{1, \mathfrak{B}}$. Our essential tools are the Calderón-Zygmund decomposition and the dyadic structure of the basis which is assumed a priori.

It is known that there are several characterizations of the Muckenhoupt class $A_{\infty, \mathfrak{Q}}$, which are defined by many authors such as Muckenhoupt, Coifman and Fefferman, Fujii and so on. However, these characterizations are not valid for a general basis $\mathfrak{B}$ instead of a basis $\mathfrak{Q}$. In the recent paper [5], Duoandikoetxea, MartínReyes and Ombrosi compared several characterizations of $A_{\infty, \mathfrak{B}}$ on a $\sigma$-finite measure space $(X, \mu)$, a basis is a collection of $\mu$-measurable subsets $B$ of $X$ such that $0<\mu(B)<\infty$. They established several implications among such conditions without further assumptions on the basis (or, for example, assuming the boundedness of the maximal operator associated with $\mathfrak{B}$ ), but their assumptions could not decide whether the weights $w \in A_{\infty, \mathfrak{B}}$ belong to $\mathrm{RH}_{1, \mathfrak{B}}$.

The paper is organized as follows: In Section 2 we state and prove two theorems (Theorems 2.6 and 2.10). For a general basis $\mathfrak{B}$, we consider the weight $w$ belonging to the reverse Hölder classes $\mathrm{RH}_{s, \mathfrak{B}}, 1<s \leq \infty$, and, for the weighted general maximal operator $M_{\mathfrak{B}, w}$, we introduce a sufficient condition for one-weight norm inequalities to hold. As a corollary (Corollary 2.7), we show that, if the general maximal operator $M_{\mathfrak{B}}$ is bounded on $L^{p}\left(\mathbf{R}^{n}\right)$ for $p>1$ and the weight $w$ belongs to the reverse Hölder class $\mathrm{RH}_{\infty, \mathfrak{B}}$, then the weighted general maximal operator $M_{\mathfrak{B}, w}$ is bounded on $L^{p}\left(\mathbf{R}^{n}, w\right)$ for $p>1$. In Section 3 we investigate some properties of $\mathrm{RH}_{\infty, \mathfrak{B}}$ weights and show how they are related to the Muckenhoupt class $A_{1, \mathfrak{B}}$. In Section 4, under the assumption that the general basis $\mathfrak{B}$ has dyadic substructure with the Stein property, we introduce the Gehring lemma (Theorem 4.6) and investigate the equivalence between the Muckenhoupt class $A_{\infty, \mathfrak{B}}$ and the reverse Hölder class $\mathrm{RH}_{1, \mathfrak{B}}$ (Theorems 4.8 and 4.10). We also discuss an equivalent way of defining the reverse Hölder class $\mathrm{RH}_{1, \mathfrak{B}}$ (Theorem 4.12) following their papers [9] and [15]. In the final section (Section 5) we extend the nice formula (1.2) to the higher dimensions (Lemma 5.1. This formula enables us that the weighted estimates for strong maximal operators and the Kakeya maximal operators in the higher dimensions (Proposition 5.3).

Throughout this paper all the notations are standard or will be defined as needed. The letter $C$ will be used for constants that may change from one occurrence to another. Constants with subscripts, such as $C_{1}, C_{2}$, do not change in different occurrences. By $A \approx B$ we mean that $c^{-1} B \leq A \leq c B$ with some positive constant $c$ independent of appropriate quantities. 


\section{General maximal operators and weight classes}

By a basis in $\mathbf{R}^{n}$ we mean a collection of open and bounded sets $\mathfrak{B}$. For a basis $\mathfrak{B}$ we consider associated weights $w$ such that $0<w(B)<\infty$ for every $B \in \mathfrak{B}$. Given such a $\mathfrak{B}$ and $w$, the corresponding maximal operator $M_{\mathfrak{B}, w}$ is defined by

$$
M_{\mathfrak{B}, w} f(x)=\sup _{B \in \mathfrak{B}} \mathbf{1}_{B}(x) \frac{1}{w(B)} \int_{B}|f(y)| w(y) d y .
$$

If $w \equiv 1$, we just write $M_{\mathfrak{B}} f(x)$. Following the notation in [4], we define $B(w)=\frac{w(B)}{|B|}$.

Definition 2.1. Let $1 \leq p<\infty$. A weight $w$ belongs to the Muckenhoupt class $A_{p, \mathfrak{B}}$ whenever its Muckenhoupt constant $[w]_{A_{p, \mathfrak{B}}}$ is finite. That is,

$$
\begin{aligned}
{[w]_{A_{p, \mathfrak{B}}} } & =\sup _{B \in \mathfrak{B}} B(w) B\left(w^{-1 /(p-1)}\right)^{p-1}<\infty, \quad 1<p<\infty, \\
{[w]_{A_{1, \mathfrak{B}}} } & =\sup _{B \in \mathfrak{B}} \frac{B(w)}{\operatorname{essinf} \inf _{x \in B} w(x)}<\infty .
\end{aligned}
$$

Notice that by Hölder's inequality, for $1<p<q<\infty$,

$$
1 \leq[w]_{A_{q, \mathfrak{B}}} \leq[w]_{A_{p, \mathfrak{B}}}<\infty
$$

and hence the inclusion $A_{p, \mathfrak{B}} \subset A_{q, \mathfrak{B}}$ holds. So, for $1<p<\infty$, the Muckenhoupt classes $A_{p, \mathfrak{B}}$ form an increasing chain and thus we define

$$
A_{\infty, \mathfrak{B}}=\bigcup_{p>1} A_{p, \mathfrak{B}}
$$

Definition 2.2. Let $1<p \leq \infty$. A weight $w$ belongs to the reverse Hölder class $\mathrm{RH}_{p, \mathfrak{B}}$ if and only if

$$
\begin{aligned}
{[w]_{\mathrm{RH}_{p, \mathfrak{B}}} } & =\sup _{B \in \mathfrak{B}} \frac{B\left(w^{p}\right)^{1 / p}}{B(w)}<\infty, \quad 1<p<\infty, \\
{[w]_{\mathrm{RH}}{ }_{\infty, \mathfrak{B}} } & =\sup _{B \in \mathfrak{B}} \frac{\operatorname{ess} \sup _{x \in B} w(x)}{B(w)}<\infty .
\end{aligned}
$$

It follows from Hölder's inequality that, for $1<p<q<\infty$,

$$
1 \leq[w]_{\mathrm{RH}_{p, \mathfrak{B}}} \leq[w]_{\mathrm{RH}_{q, \mathfrak{B}}}<\infty
$$

and hence that the inclusion $\mathrm{RH}_{p, \mathfrak{B}} \supset \mathrm{RH}_{q, \mathfrak{B}}$ holds. So, for $1<p<\infty$, the reverse Hölder classes $\mathrm{RH}_{p, \mathfrak{B}}$ form a decreasing chain and thus we define

$$
\mathrm{RH}_{1, \mathfrak{B}}=\bigcup_{p>1} \mathrm{RH}_{p, \mathfrak{B}}
$$

For the general bases $\mathfrak{B}$ and associated weights $w$, very little is known concerning the boundedness of $M_{\mathfrak{B}}$ and $M_{\mathfrak{B}, w}$ on $L^{p}(w)$. The most important result of one weight theory for general maximal operators is theorem due to Jawerth [10]. This theorem was reproved by Lerner [11] with better understanding of the dependency of the constants.

Theorem 2.3. (Jawerth [10]) Let $1<p<\infty$. For the general bases $\mathfrak{B}$ and associated weights $w$, let $\sigma=w^{-1 /(p-1)}$. Then $M_{\mathfrak{B}}$ is bounded on $L^{p}(w)$ and on $L^{p^{\prime}}(\sigma)$ if and only if $w \in A_{p, \mathfrak{B}}, M_{\mathfrak{B}, \sigma}$ is bounded on $L^{p}(\sigma)$ and $M_{\mathfrak{B}, w}$ is bounded on $L^{p^{\prime}}(w)$. 
The following abstract theorem from [14], which is proved by the use of Theorem 2.3, gives a necessary and sufficient condition for the boundedness of the $M_{\mathfrak{B}, w}$, in terms of the unweighted maximal function $M_{\mathfrak{B}}$ in the special case $w \in A_{\infty, \mathfrak{B}}$.

Theorem 2.4. (Pérez [14]) The following are equivalent:

(i) For every $1<p<\infty$ and every $w \in A_{p, \mathfrak{B}}$, we have

$$
M_{\mathfrak{B}}: L^{p}(w) \rightarrow L^{p}(w)
$$

(ii) For every $1<p<\infty$ and every $w \in A_{\infty, \mathfrak{B}}$, we have

$$
M_{\mathfrak{B}, w}: L^{p}(w) \rightarrow L^{p}(w) .
$$
inition.

Theorem 2.4 and some important examples (cf. [14]) motivate the following def-

Definition 2.5. A basis $\mathfrak{B}$ is a Muckenhoupt basis if for every $1<p<\infty$ and every $w \in A_{p, \mathfrak{B}}$, we have

$$
M_{\mathfrak{B}}: L^{p}(w) \rightarrow L^{p}(w)
$$

With this definition Theorem 2.4 states that $\mathfrak{B}$ is a Muckenhoupt basis if and only if the weighted maximal function satisfies $M_{\mathfrak{B}, w}: L^{p}(w) \rightarrow L^{p}(w)$ for every $1<p<\infty$ and every $w \in A_{\infty, \mathfrak{B}}$.

We are interested in the theory of weights for the Kakeya maximal operators. The main interest of this theory is to determine the factor $N$ appearing in its operator norms. From this point of view, Theorem 2.4 is quite abstract and thus it cannot apply to our situation. Attempting one weighted estimate for the Kakeya maximal operator $M_{\mathfrak{K}_{N}}$ in Wolff's range (cf. [21]), we found the following theorem concerning general maximal operators which is our first theorem of this paper.

Theorem 2.6. Let $\mathfrak{B}$ be a basis and $1<p, s \leq \infty$. Suppose that $w \in \mathrm{RH}_{s, \mathfrak{B}}$ with $[w]_{\mathrm{RH}_{s, \mathfrak{B}}}=C_{1},\left\|M_{\mathfrak{B}}\right\|_{L^{t}\left(\mathbf{R}^{n}\right)}=C_{2}$ with $t=p-(p-1) / s>1$. Suppose further that there exists a constant $C_{3}>0$ such that

$$
\sum_{j} \frac{w\left(E\left(B_{j}\right)\right)}{B_{j}(w)} \leq C_{3}\left|\bigcup_{j} B_{j}\right|
$$

holds for any countable subfamily $\left\{B_{j}\right\} \subset \mathfrak{B}$ and any pairwise disjoint subset $E\left(B_{j}\right) \subset B_{j}$ for varying $j$. Then we have

$$
\left\|M_{\mathfrak{B}, w}\right\|_{L^{p}(w)} \leq 2 C_{1}^{1 / p^{\prime}} C_{2}^{1 /\left(p^{\prime} s\right)^{\prime}} C_{3}^{1 / p}
$$

Proof. Let $f \in L^{p}(w)$. For every integer $k$, we shall consider the set

$$
S_{k}=\left\{x \in \mathbf{R}^{n}: 2^{k}<M_{\mathfrak{B}, w} f(x) \leq 2^{k+1}\right\} .
$$

Using a limiting argument, we may assume that $S_{k}$ are compact sets. From the definition of $M_{\mathfrak{B}, w}, S_{k} \subset \bigcup_{j} B_{k, j}$, where $B_{k, j} \in \mathfrak{B}$ satisfies

$$
\frac{1}{w\left(B_{k, j}\right)} \int_{B_{k, j}}|f(y)| w(y) d y>2^{k} \text {. }
$$

Define $E\left(B_{k, 1}\right)=B_{k, 1} \cap S_{k}$ and, for $j>1$,

$$
E\left(B_{k, j}\right)=\left(B_{k, j} \backslash \bigcup_{i<j} B_{k, i}\right) \cap S_{k} .
$$


The sets $S_{k}$ form a disjoint collection and each $S_{k}$ is the disjoint union of the sets $E\left(B_{k, j}\right)$ for varying $j$. Thus,

$$
\begin{aligned}
\int_{\mathbf{R}^{n}} M_{\mathfrak{B}, w} f(x)^{p} w(x) d x & =\sum_{k, j} \int_{E\left(B_{k, j}\right)} M_{\mathfrak{B}, w} f(x)^{p} w(x) d x \leq \sum_{k, j} 2^{(k+1) p} w\left(E\left(B_{k, j}\right)\right) \\
& \leq 2^{p} \sum_{k, j} w\left(E\left(B_{k, j}\right)\right)\left(\frac{1}{w\left(B_{k, j}\right)} \int_{B_{k, j}}|f(y)| w(y) d y\right)^{p} .
\end{aligned}
$$

Notice that

$$
\left(\frac{1}{w\left(B_{k, j}\right)} \int_{B_{k, j}}|f(y)| w(y) d y\right)^{p}=B_{k, j}(w)^{-p}\left(\frac{1}{\left|B_{k, j}\right|} \int_{B_{k, j}}|f(y)| w(y) d y\right)^{p}
$$

and by Hölder's inequality that

$$
\begin{aligned}
& \left(\frac{1}{\left|B_{k, j}\right|} \int_{B_{k, j}}|f(y)| w(y) d y\right)^{p}=\left(\frac{1}{\left|B_{k, j}\right|} \int_{B_{k, j}}|f(y)| w(y)^{1 / p} \cdot w(y)^{1 / p^{\prime}} d y\right)^{p} \\
& \leq\left(\frac{1}{\left|B_{k, j}\right|} \int_{B_{k, j}}|f(y)|^{p / t} w(y)^{1 / t} d y\right)^{t}\left(\frac{1}{\left|B_{k, j}\right|} \int_{B_{k, j}} w(y)^{s} d y\right)^{p /\left(p^{\prime} s\right)},
\end{aligned}
$$

where we have used

$$
\frac{p}{t}=\frac{p}{p-(p-1) / s}=\frac{1}{1-1 /\left(p^{\prime} s\right)}=\frac{p^{\prime} s}{p^{\prime} s-1}=\left(p^{\prime} s\right)^{\prime} .
$$

These yield

$$
\begin{aligned}
& \int_{\mathbf{R}^{n}} M_{\mathfrak{B}, w} f(x)^{p} w(x) d x \\
& \leq 2^{p} \sum_{k, j} \frac{w\left(E\left(B_{k, j}\right)\right)}{B_{k, j}(w)} B_{k, j}(w)^{1-p}\left(B_{k, j}\left(w^{s}\right)^{1 / s}\right)^{p-1}\left(\frac{1}{\left|B_{k, j}\right|} \int_{B_{k, j}}|f(y)|^{p / t} w(y)^{1 / t} d y\right)^{t} \\
& \leq 2^{p} C_{1}^{p-1} \sum_{k, j} \mu_{k, j} g_{k, j},
\end{aligned}
$$

where

$$
\mu_{k, j}=\frac{w\left(E\left(B_{k, j}\right)\right)}{B_{k, j}(w)}, \quad g_{k, j}=\left(\frac{1}{\left|B_{k, j}\right|} \int_{B_{k, j}}|f(y)|^{p / t} w(y)^{1 / t} d y\right)^{t} .
$$

We view the sum $\sum_{k, j} \mu_{k, j} g_{k, j}$ as an integral on a measure space $(X, \mu)$ built over the set $X=\{(k, j)\}$ by assigning to each $(k, j)$ the measure $\mu_{k, j}$. For $\lambda>0$ call

$$
\Gamma(\lambda)=\left\{(k, j) \in X: g_{k, j}>\lambda\right\}, \quad G(\lambda)=\bigcup_{(k, j) \in \Gamma(\lambda)} B_{k, j} .
$$

Then $\sum_{k, j} \mu_{k, j} g_{k, j}=\int_{0}^{\infty} \mu(\Gamma(\lambda)) d \lambda$. Observe that

$$
\mu(\Gamma(\lambda))=\sum_{(k, j) \in \Gamma(\lambda)} \frac{w\left(E\left(B_{k, j}\right)\right)}{B_{k, j}(w)} \leq C_{3}|G(\lambda)|
$$

and that

$$
G(\lambda) \subset\left\{x \in \mathbf{R}^{n}: M_{\mathfrak{B}}\left[|f|^{p / t} w^{1 / t}\right](x)^{t}>\lambda\right\}
$$


These entail

$$
\begin{aligned}
\int_{\mathbf{R}^{n}} M_{\mathfrak{B}, w} f(x)^{p} w(x) d x & \leq 2^{p} C_{1}^{p-1} C_{3} \int_{\mathbf{R}^{n}} M_{\mathfrak{B}}\left[|f|^{p / t} w^{1 / t}\right](x)^{t} d x \\
& \leq 2^{p} C_{1}^{p-1} C_{3} C_{2}^{t} \int_{\mathbf{R}^{n}}|f(x)|^{p} w(x) d x .
\end{aligned}
$$

Consequently,

$$
\left\|M_{\mathfrak{B}, w}\right\|_{L^{p}(w)} \leq 2 C_{1}^{1 / p^{\prime}} C_{2}^{1 /\left(p^{\prime} s\right)^{\prime}} C_{3}^{1 / p}
$$

This completes the proof.

The next corollary leads the relations (1.3) and (1.4).

Corollary 2.7. Let $\mathfrak{B}$ be a basis and $1<p \leq \infty$. Suppose that the weight $w$ is in $\mathrm{RH}_{\infty, \mathfrak{B}}$ with $[w]_{\mathrm{RH}_{\infty, \mathfrak{B}}}=C_{1}$ and that $\left\|M_{\mathfrak{B}}\right\|_{L^{p}\left(\mathbf{R}^{n}\right)}=C_{2}$. Then we have

$$
\left\|M_{\mathfrak{B}, w}\right\|_{L^{p}(w)} \leq 2 C_{1} C_{2} .
$$

Proof. Since $t=p$ and $\left(p^{\prime} s\right)^{\prime}=1$, we merely check the condition (2.1) with $C_{3}=C_{1}$. There holds

$$
\sum_{j} \frac{w\left(E\left(B_{j}\right)\right)}{B_{j}(w)} \leq \sum_{j}\left|E\left(B_{j}\right)\right| \frac{\operatorname{ess~sup}_{x \in B_{j}} w(x)}{B_{j}(w)} \leq C_{1} \sum_{j}\left|E\left(B_{j}\right)\right| \leq C_{1}\left|\bigcup_{j} B_{j}\right|,
$$

where we have used that the sets $E\left(B_{j}\right) \subset B_{j}$ are pairwise disjoint.

Remark 2.8. Let $w(x)=w_{0}(|x|), x \in \mathbf{R}^{2}$, and $w_{0}$ satisfy (1.1). Then by (1.2) we see that $w$ belongs to $\mathrm{RH}_{\infty, \mathfrak{K}_{N}}$. Thus, Corollary 2.7 yields (1.3) and (1.4) together with well-known unweighted estimates; see [3, 16].

When $s=\infty$ the sufficient condition (2.1) in Theorem 2.6 can be checked quite easily. But, when $1<s<\infty$, we cannot say any more. The next theorem (Theorem 2.10) characterizes the boundedness of $M_{\mathfrak{B}, w}$ on $L^{p}(w)$ in terms of a so-called Tauberian condition.

Definition 2.9. We say that a weight $w$ associated to the basis $\mathfrak{B}$ satisfies the Tauberian condition (A) if there are constants $0<\lambda<1,0<c=c(\lambda)<\infty$ such that for all measurable sets $E$

$$
w\left(\left\{x \in \mathbf{R}^{n}: M_{\mathfrak{B}}\left(\mathbf{1}_{E}\right)(x)>\lambda\right\}\right) \leq c w(E) .
$$

Theorem 2.10. Let $\mathfrak{B}$ be a basis. Let $w \in \mathrm{RH}_{s, \mathfrak{B}}, 1<s \leq \infty$, and $w$ satisfy the condition (A). Suppose that $M_{\mathfrak{B}}: L^{p}\left(\mathbf{R}^{n}\right) \rightarrow L^{p}\left(\mathbf{R}^{n}\right)$ for all $p>1$. Then $M_{\mathfrak{B}, w}: L^{p}(w) \rightarrow L^{p}(w)$ holds for all $p>1$.

Proof. Let $f \in L^{p}(w)$. In the same manner as in the proof of Theorem 2.6, for every integer $k$, we consider the set

$$
S_{k}=\left\{x \in \mathbf{R}^{n}: 2^{k}<M_{\mathfrak{B}, w} f(x) \leq 2^{k+1}\right\} .
$$

We choose compact sets $K_{k} \subset S_{k}$ and, for each $k$, we choose a finite cover $\left\{B_{k, j}\right\} \subset \mathfrak{B}$ such that

$$
K_{k} \subset \bigcup_{j} B_{k, j} \quad \text { and } \quad \frac{1}{w\left(B_{k, j}\right)} \int_{B_{k, j}}|f(y)| w(y) d y>2^{k} .
$$

Now, we have

$$
\int_{\bigcup_{k} K_{k}} M_{\mathfrak{B}, w} f(x)^{p} w(x) d x \leq 2^{p} \sum_{k} 2^{k p} w\left(\bigcup_{j} B_{k, j}\right) .
$$


We claim that then there exists a subfamily $\left\{\bar{B}_{k, j}\right\} \subset\left\{B_{k, j}\right\}$ satisfying

$$
\sum_{k} 2^{k p} w\left(\bigcup_{j} B_{k, j}\right) \leq C \sum_{k} 2^{k p} w\left(\bigcup_{j} \bar{B}_{k, j}\right)
$$

and, for each $(k, j)$, there exists a subset $E\left(\bar{B}_{k, j}\right) \subset \bar{B}_{k, j}$ such that

$$
(1-\lambda)\left|\bar{B}_{k, j}\right| \leq\left|E\left(\bar{B}_{k, j}\right)\right|
$$

and the sets $E\left(\bar{B}_{k, j}\right)$ are pairwise disjoint for varying $(k, j)$.

We shall verify this claim. Without loss of generality we may assume that $B_{k, j}=$ $\emptyset$ except for a finite number of $(k, j)$. Let $N=\max \left\{k: B_{k, j} \neq \emptyset\right\}$. We follow a well-known selecting procedure argument (cf. [6, p. 463] for instance).

Step 1. Let $\bar{B}_{N, 1}=B_{N, 1}$ and, once $\bar{B}_{N, 1}, \ldots, \bar{B}_{N, j-1}$ have been selected, we choose $\bar{B}_{N, j}$ to be the first set in $\left\{B_{N, \cdot}\right\}$ (if any) such that

$$
\left|\bar{B}_{N, j} \cap\left(\bigcup_{s<j} \bar{B}_{N, s}\right)\right|<\lambda\left|\bar{B}_{N, j}\right| .
$$

Let $E_{N}=\bigcup_{j} \bar{B}_{N, j}$. Now we verify that

$$
\bigcup_{j} B_{N, j} \subset\left\{x \in \mathbf{R}^{n}: M_{\mathfrak{B}}\left[\mathbf{1}_{E_{N}}\right](x) \geq \lambda\right\} .
$$

Let $x \in \bigcup_{j} B_{N, j}$. If $x \in E_{N}$, then it is obvious that it is contained on the set to the right since $\lambda<1$. If $x \notin E_{N}$, then $x$ is contained in some discarded $B$ in the selection process and hence $\left|B \cap E_{N}\right| \geq \lambda|B|$, which means that $M_{\mathfrak{B}}\left[\mathbf{1}_{E_{N}}\right](x) \geq \lambda$. Since $w$ satisfies the condition (A), (2.6) yields

$$
w\left(\bigcup_{j} B_{N, j}\right) \leq C w\left(E_{N}\right) .
$$

If we let $E\left(\bar{B}_{N, j}\right)=\bar{B}_{N, j} \backslash \bigcup_{s<j} \bar{B}_{N, s}$, then we see that, by $(2.5),\left\{E\left(\bar{B}_{N, j}\right)\right\}$ is a disjoint family with $E\left(\bar{B}_{N, j}\right) \subset \bar{B}_{N, j}$ and $(1-\lambda)\left|\bar{B}_{N, j}\right|<\left|E\left(\bar{B}_{N, j}\right)\right|$.

Step 2. Let $\bar{B}_{N-1,1}$ be the first set in $\left\{B_{N-1,}\right\}$ such that

$$
\left|\bar{B}_{N-1,1} \cap E_{N}\right|<\lambda\left|\bar{B}_{N-1,1}\right| \text {. }
$$

Once $\bar{B}_{N-1,1}, \ldots, \bar{B}_{N-1, j-1}$ have been selected, we choose $\bar{B}_{N-1, j}$ to be the first set in $\left\{B_{N-1,1}\right\}$ (if any) such that

$$
\left|\bar{B}_{N-1, j} \cap\left\{E_{N} \cup\left(\bigcup_{s<j} \bar{B}_{N-1, s}\right)\right\}\right|<\lambda\left|\bar{B}_{N-1, j}\right| .
$$

Let $E_{N-1}=\bigcup_{j} \bar{B}_{N-1, j}$. By the same observation as the above, we can verify that

$$
\bigcup_{j} B_{N-1, j} \subset\left\{x \in \mathbf{R}^{n}: M_{\mathfrak{B}}\left[\mathbf{1}_{E_{N-1} \cup E_{N}}\right](x) \geq \lambda\right\}
$$

and see that

$$
w\left(\bigcup_{j} B_{N-1, j}\right) \leq C w\left(E_{N-1} \cup E_{N}\right) .
$$


If we let

$$
E\left(\bar{B}_{N-1, j}\right)=\bar{B}_{N, j} \backslash\left\{E_{N} \cup\left(\bigcup_{s<j} \bar{B}_{N-1, s}\right)\right\}
$$

then we have, by (2.7), $\left\{E\left(\bar{B}_{N-1, j}\right)\right\} \cup\left\{E\left(\bar{B}_{N, j}\right)\right\}$ is a disjoint family with $E\left(\bar{B}_{N-1, j}\right) \subset$ $\bar{B}_{N-1, j}$ and $(1-\lambda)\left|\bar{B}_{N-1, j}\right|<\left|E\left(\bar{B}_{N-1, j}\right)\right|$.

Continuing these steps, We obtain a subfamily $\left\{\bar{B}_{k, j}\right\} \subset\left\{B_{k, j}\right\}$ satisfying (2.4) and

$$
w\left(\bigcup_{j} B_{k, j}\right) \leq C w\left(\bigcup_{\ell=k}^{N} E_{\ell}\right)
$$

where

$$
E_{\ell}=\bigcup_{j} \bar{B}_{\ell, j}
$$

It follows that

$$
\begin{aligned}
\sum_{k} 2^{k p} w\left(\bigcup_{j} B_{k, j}\right) & \leq C \sum_{k} 2^{k p} w\left(\bigcup_{\ell=k}^{N} E_{\ell}\right) \leq C \sum_{k} 2^{k p}\left(\sum_{\ell=k}^{N} w\left(E_{\ell}\right)\right) \\
& =C \sum_{k}\left(\sum_{\ell \leq k} 2^{\ell p}\right) w\left(E_{k}\right) \leq C \sum_{k} 2^{k p} w\left(E_{k}\right) \\
& =C \sum_{k} 2^{k p} w\left(\bigcup_{j} \bar{B}_{k, j}\right),
\end{aligned}
$$

which is exactly (2.3).

There holds by (2.2) and (2.3)

$$
\begin{aligned}
\int_{\bigcup_{k} K_{k}} M_{\mathfrak{B}, w} f(x)^{p} w(x) d x & \leq C \sum_{k} 2^{k p} w\left(\bigcup_{j} B_{k, j}\right) \leq C \sum_{k} 2^{k p} w\left(\bigcup_{j} \bar{B}_{k, j}\right) \\
& \leq C \sum_{k, j} w\left(\bar{B}_{k, j}\right)\left(\frac{1}{w\left(\bar{B}_{k, j}\right)} \int_{\bar{B}_{k, j}}|f(y)| w(y) d y\right)^{p} .
\end{aligned}
$$

Recalling $t=p-(p-1) / s$,

$$
\left(\frac{1}{w\left(\bar{B}_{k, j}\right)} \int_{\bar{B}_{k, j}}|f(y)| w(y) d y\right)^{p} \leq C \frac{1}{\bar{B}_{k, j}(w)}\left(\frac{1}{\left|\bar{B}_{k, j}\right|} \int_{\bar{B}_{k, j}}|f(y)|^{p / t} w(y)^{1 / t} d y\right)^{t}
$$

Thus, by (2.4),

$$
\begin{aligned}
\int_{\bigcup_{k} K_{k}} M_{\mathfrak{B}, w} f(x)^{p} w(x) d x & \leq C \sum_{k, j}\left|\bar{B}_{k, j}\right|\left(\frac{1}{\left|\bar{B}_{k, j}\right|} \int_{\bar{B}_{k, j}}|f(y)|^{p / t} w(y)^{1 / t} d y\right)^{t} \\
& \leq C \sum_{k, j}\left|E\left(\bar{B}_{k, j}\right)\right|\left(\frac{1}{\left|\bar{B}_{k, j}\right|} \int_{\bar{B}_{k, j}}|f(y)|^{p / t} w(y)^{1 / t} d y\right)^{t} \\
& \leq C \sum_{k, j} \int_{E\left(\bar{B}_{k, j}\right)} M_{\mathfrak{B}}\left[|f|^{p / t} w^{1 / t}\right](x)^{t} d x \\
& \leq C \int_{\mathbf{R}^{n}} M_{\mathfrak{B}}\left[|f|^{p / t} w^{1 / t}\right](x)^{t} d x \leq C \int_{\mathbf{R}^{n}}|f(x)|^{p} w(x) d x,
\end{aligned}
$$


where we have used the sets $E\left(\bar{B}_{k, j}\right)$ are pairwise disjoint. This completes the proof.

Remark 2.11. The weighted Tauberian condition for general maximal operators appears in $[10,13]$, for instance. It is shown in [8] that the condition $(\mathrm{A})$ is equivalent to $M_{\mathfrak{B}}: L^{p}(w) \rightarrow L^{p}(w)$ for sufficient large $p$ provided that $\mathfrak{B}$ is a homothecy invariant basis consisting of convex sets.

\section{The class $\mathrm{RH}_{\infty, \mathfrak{B}}$}

In this section, following [4], we show some properties of $\mathrm{RH}_{\infty, \mathfrak{B}}$ weights. Throughout this section we fix a general basis $\mathfrak{B}$. We need some lemmas.

Lemma 3.1. Let $1<p, s<\infty$. A weight $w$ is in $A_{p, \mathfrak{B}} \cap \mathrm{RH}_{s, \mathfrak{B}}$ if and only if $w^{s}$ is in $A_{q, \mathfrak{B}}$ where $q=s(p-1)+1$.

Proof. Suppose that $w \in A_{p, \mathfrak{B}} \cap \mathrm{RH}_{s, \mathfrak{B}}$. Then, noticing $q-1=s(p-1)$, we have

$$
\begin{aligned}
B\left(w^{s}\right) B\left(\left(w^{s}\right)^{-1 /(q-1)}\right)^{q-1} & =B\left(w^{s}\right) B\left(w^{-1 /(p-1)}\right)^{s(p-1)} \\
& \leq C\left\{B(w) B\left(w^{-1 /(p-1)}\right)^{p-1}\right\}^{s}<\infty,
\end{aligned}
$$

where we have used $B\left(w^{s}\right) \leq C B(w)^{s}$.

Suppose, conversely, that $w^{s} \in A_{q, \mathfrak{B}}$ with $q=s(p-1)+1$. We have then

$$
B\left(w^{s}\right) B\left(w^{-1 /(p-1)}\right)^{s(p-1)} \leq C .
$$

Noticing $B(w)^{s} \leq B\left(w^{s}\right)$,

$$
B(w) B\left(w^{-1 /(p-1)}\right)^{p-1} \leq\left\{B\left(w^{s}\right) B\left(w^{-1 /(p-1)}\right)^{s(p-1)}\right\}^{1 / s} \leq C^{1 / s} .
$$

Thus, $w \in A_{p, \mathfrak{B}}$. It follows from (3.1) that

$$
B\left(w^{s}\right)^{1 / s} \leq C B\left(w^{-1 /(p-1)}\right)^{1-p} \leq C B(w),
$$

where we have used $1 \leq B(w) B\left(w^{-1 /(p-1)}\right)^{p-1}$.

Lemma 3.2. Let $p \geq 2$. If $u \in A_{p, \mathfrak{B}}$ and $v \in A_{p^{\prime}, \mathfrak{B}}$, then there exists a constant $C>0$ such that, for every $B \in \mathfrak{B}$,

$$
B(u)^{1 / p} B(v)^{1 / p^{\prime}} \leq C B\left(u^{1 / p} v^{1 / p^{\prime}}\right) .
$$

Proof. Fix a $B \in \mathfrak{B}$. Then there is a constant $C$ such that

$$
B(u) B\left(u^{1-p^{\prime}}\right)^{p-1} \leq C, \quad B(v) B\left(v^{1-p}\right)^{p^{\prime}-1} \leq C .
$$

Raise the first equation to the power $1 / p$ and the second to the power $1 / p^{\prime}$ and multiply them together. Then after re-arranging terms we get

$$
B(u)^{1 / p} B(v)^{1 / p^{\prime}} \leq C B\left(u^{1-p^{\prime}}\right)^{-1 / p^{\prime}} B\left(v^{1-p}\right)^{-1 / p} .
$$

Since $p / p^{\prime}=p-1$ and $p^{\prime} / p=p^{\prime}-1$, by Hölder's inequality

$$
B\left(u^{-1 / p} v^{-1 / p^{\prime}}\right) \leq B\left(u^{1-p^{\prime}}\right)^{1 / p^{\prime}} B\left(v^{1-p}\right)^{1 / p} .
$$

Therefore, by combining these two inequalities we see that

$$
B(u)^{1 / p} B(v)^{1 / p^{\prime}} \leq C B\left(u^{-1 / p} v^{-1 / p^{\prime}}\right)^{-1} .
$$

By the use of the inequality $1 \leq B(f) B\left(f^{1-p^{\prime}}\right)^{p-1}$,

$$
B\left(u^{-1 / p} v^{-1 / p^{\prime}}\right)^{-1} \leq B\left(\left(u^{-1 / p} v^{-1 / p^{\prime}}\right)^{1-p^{\prime}}\right)^{p-1}=B\left(\left(u^{1 / p} v^{1 / p^{\prime}}\right)^{p^{\prime}-1}\right)^{p-1} .
$$


Noticing $p \geq 2$, by applying Hölder's inequality the above expression can be bounded above by $B\left(u^{1 / p} v^{1 / p^{\prime}}\right)$. Therefore (3.2) holds.

Lemma 3.3. Let $1<p<\infty$. If $u \in A_{\infty, \mathfrak{B}} \cap \mathrm{RH}_{p, \mathfrak{B}}$ and $v \in A_{\infty, \mathfrak{B}} \cap \mathrm{RH}_{p^{\prime}, \mathfrak{B}}$, then there exists a constant $C$ such that, for every $B \in \mathfrak{B}$,

$$
B\left(u^{p}\right)^{1 / p} B\left(v^{p^{\prime}}\right)^{1 / p^{\prime}} \leq C B(u v) .
$$

Proof. By the inclusion property of the class $A_{p, \mathfrak{B}}$ we may assume that

$$
u \in A_{q_{1}, \mathfrak{B}}, \quad v \in A_{r_{1}, \mathfrak{B}} \quad \text { with } \quad p\left(r_{1}-1\right)=p^{\prime}\left(q_{1}-1\right)=s>1 .
$$

Since $u \in \mathrm{RH}_{p, \mathfrak{B}}$ and $v \in \mathrm{RH}_{p^{\prime}, \mathfrak{B}}$, then, by Lemma 3.1,

$$
u^{p} \in A_{q, \mathfrak{B}}, \quad v^{p^{\prime}} \in A_{r, \mathfrak{B}} \quad \text { with } \quad q=p\left(q_{1}-1\right)+1, \quad r=p^{\prime}\left(r_{1}-1\right)+1 .
$$

Since $q=s(p-1)$ and $r=s\left(p^{\prime}-1\right)$, by Lemma 3.1 again, this is equivalent to

$$
u^{p / s} \in A_{p, \mathfrak{B}} \cap \mathrm{RH}_{s, \mathfrak{B}}, \quad v^{p^{\prime} / s} \in A_{p^{\prime}, \mathfrak{B}} \cap \mathrm{RH}_{s, \mathfrak{B}} .
$$

This relation and Lemma 3.2 yield

$$
B\left(u^{p}\right)^{1 / s p} B\left(v^{p^{\prime}}\right)^{1 / s p^{\prime}} \leq C B\left(u^{p / s}\right)^{1 / p} B\left(v^{p^{\prime} / s}\right)^{1 / p^{\prime}} \leq C B\left((u v)^{1 / s}\right) \leq C B(u v)^{1 / s} .
$$

If we raise both sides to the power $s$, we get inequality (3.3).

Theorem 3.4. If the weight $w$ is in $\mathrm{RH}_{\infty, \mathfrak{B}}$, then $w^{r}$ is in $\mathrm{RH}_{\infty, \mathfrak{B}}$ for $r \geq 1$. If the weight $w$ is in $A_{\infty, \mathfrak{B}} \cap \mathrm{RH}_{\infty, \mathfrak{B}}$, then $w^{r}$ is in $\mathrm{RH}_{\infty, \mathfrak{B}}$ for $0<r<1$.

Proof. Let $r \geq 1$. Hölder's inequality gives, for any $B \in \mathfrak{B}$ and any $x \in B$,

$$
w(x)^{r} \leq C B(w)^{r} \leq C B\left(w^{r}\right)
$$

Let $0<r<1$. Assuming $w \in A_{q, \mathfrak{B}}$ for some large $q>1$ we have by Lemma 3.1

$$
w^{r} \in A_{p, \mathfrak{B}} \cap \mathrm{RH}_{\mathfrak{B}, 1 / r} \quad \text { with } \quad q=\frac{1}{r}(p-1)+1 \quad \text { and } \quad p>1 .
$$

Thus,

$$
w(x)^{r} \leq C B(w)^{r} \leq C B\left(w^{r}\right) .
$$

Theorem 3.5. If $u$ and $v$ belong to $A_{\infty, \mathfrak{B}} \cap \mathrm{RH}_{\infty, \mathcal{B}}$, then $u v$ belongs to $\mathrm{RH}_{\infty, \mathfrak{B}}$.

Proof. Fix a $B \in \mathfrak{B}$ and an $x \in B$. Then

$$
u(x) v(x) \leq C B(u) B(v) \leq C B\left(u^{2}\right)^{1 / 2} B\left(v^{2}\right)^{1 / 2} .
$$

Since $u$ and $v$ belong to $A_{\infty, \mathfrak{B}} \cap \mathrm{RH}_{2, \mathfrak{B}}$, by Lemma 3.3 , the above expression can be bounded above by $C B(u v)$. This means that $u v \in \mathrm{RH}_{\infty, \mathfrak{B}}$.

Theorem 3.6. Fix $p>1$. If $w$ is in $A_{1, \mathfrak{B}}$, then $w^{1-p}$ is in $A_{p, \mathfrak{B}} \cap \mathrm{RH}_{\infty, \mathfrak{B}}$. Conversely, if $w$ is in $A_{p, \mathfrak{B}} \cap \mathrm{RH}_{\infty, \mathfrak{B}}$, then $w^{1-p^{\prime}}$ is in $A_{1, \mathfrak{B}}$.

Proof. That $w \in A_{1, \mathfrak{B}}$ implies $w \in A_{p^{\prime}, \mathfrak{B}}$. So, $w^{1-p} \in A_{p, \mathfrak{B}}$. It follows for $B \in \mathfrak{B}$ that

$$
1 \leq B(w) B\left(w^{1-p}\right)^{1 /(p-1)} \leq B\left(w^{1-p}\right)^{1 /(p-1)} \underset{x \in B}{\operatorname{essinf}} w(x)
$$

and hence

$$
\underset{x \in B}{\operatorname{ess} \sup } w(x)^{1-p} \leq B\left(w^{1-p}\right) .
$$

This means that $w^{1-p} \in A_{p, \mathfrak{B}} \cap \mathrm{RH}_{\infty, \mathfrak{B}}$. Conversely, if $w \in A_{p, \mathfrak{B}} \cap \mathrm{RH}_{\infty, \mathfrak{B}}$, then, for $B \in \mathfrak{B}$,

$$
\underset{x \in B}{\operatorname{ess} \sup } w(x) B\left(w^{1-p^{\prime}}\right)^{p-1} \leq C B(w) B\left(w^{1-p^{\prime}}\right)^{p-1} \leq C<\infty .
$$


This implies

$$
B\left(w^{1-p^{\prime}}\right) \leq C \underset{x \in B}{\operatorname{essinf}} w(x)^{1-p^{\prime}},
$$

which means that $w^{1-p^{\prime}} \in A_{1, \mathfrak{B}}$.

Remark 3.7. Suppose that $M_{\mathfrak{B}}: L^{p}\left(\mathbf{R}^{n}\right) \rightarrow L^{p}\left(\mathbf{R}^{n}\right)$ for some $p>1$. Then, using the well-known Rubio de Francia algorithm, one can produce many $A_{1, \mathfrak{B}^{-}}$weights. Theorem 3.6 asserts that, if $w$ is in $A_{1, \mathfrak{B}}$, then $w^{-1}$ is in $A_{\infty, \mathfrak{B}} \cap \mathrm{RH}{ }_{\infty, \mathfrak{B}}$. Hence, we have many weights belonging to $A_{\infty, \mathfrak{B}} \cap \mathrm{RH}_{\infty, \mathfrak{B}}$.

\section{The classes $A_{\infty, \mathfrak{B}}$ and $\mathrm{RH}_{1, \mathfrak{B}}$}

In this section we discuss the equivalence between the classes $A_{\infty, \mathfrak{B}}$ and $\mathrm{RH}_{1, \mathfrak{B}}$ when the basis $\mathfrak{B}$ has dyadic substructure with the Stein property. The following definition expresses the relevant property of a basis needed for our purposes. In the case when $\mathfrak{B}=\mathfrak{D}$, the usual collection of dyadic cubes in $\mathbf{R}^{n}$, it was obtained by Stein in [20].

Definition 4.1. We say that a basis $\mathfrak{B}$ satisfies the Stein property if and only if there exists a constant $c$ such that, for any non-negative function $f \in L_{\text {loc }}^{1}\left(\mathbf{R}^{n}\right)$, $B \in \mathfrak{B}$ and $\lambda>B(f)$, we have

$$
\int_{\{x \in B: f(x)>\lambda\}} f(x) d x \leq c \lambda\left|\left\{x \in B: M_{\mathfrak{B}}\left[f \mathbf{1}_{B}\right](x)>\lambda\right\}\right| .
$$

Under the assumption of the Stein property, Lerner and Ombrosi established the self-improving property of general maximal operators on quasi-Banach function spaces [12].

Definition 4.2. We say that the basis $\mathfrak{B}$ has a dyadic substructure if and only if, for all $B \in \mathfrak{B}$, there exists a dyadic subset $\mathfrak{D}(B) \subset \mathfrak{B}$ that satisfies the following:

(1) If $Q, R \in \mathfrak{D}(B)$ then $Q \cap R \in\{Q, R, \emptyset\}$;

(2) $B \in \mathfrak{D}(B)$ and $B$ is maximal in $\mathfrak{D}(B)$ with respect to inclusion.

The family $\mathfrak{D}(B)$ is referred to as a dyadic basis. We further assume that the basis $\mathfrak{D}(B)$ is unique and it is a density basis. That is, for all $B \in \mathfrak{B}$ and any $P \in \mathfrak{D}(B)$, we have $\mathfrak{D}(P)=\{P \cap Q: Q \in \mathfrak{D}(B)\}$. The basis $\mathfrak{D}(B)$ is called a density basis if it differentiates $L^{\infty}(B)$, namely, if for every $f \in L^{\infty}(B)$ and for almost every $x \in B$, there exists a sequence $\left\{Q_{k}\right\} \subset \mathfrak{D}(B)$ such that $Q_{k} \ni x$ and $\lim _{k \rightarrow \infty} Q_{k}(f)=f(x)$.

Definition 4.3. We say that the basis $\mathfrak{B}$ has dyadic substructure with the Stein property if and only if $\mathfrak{B}$ has dyadic substructure and each basis $\mathfrak{D}(B)$ satisfies the Stein property with uniform constant $c$ for varying $B \in \mathfrak{B}$.

We first verify some lemmas.

Lemma 4.4. Suppose that the basis $\mathfrak{B}$ has dyadic substructure with the Stein property. Let $f$ be a non-negative, locally integrable function on $\mathbf{R}^{n}$ and $B \in \mathfrak{B}$. Let $P, Q \in \mathfrak{D}(B)$ such that $P \subset Q$. Assume that

$$
P(f)>\lambda \text { and } Q(f) \leq \lambda .
$$

Then $\lambda<P(f) \leq c \lambda$.

Proof. We shall apply the Stein property to $Q, g \equiv P(f) \mathbf{1}_{P}$ and $\lambda$. This is justified by the fact that

$$
Q(g)=Q\left(P(f) \mathbf{1}_{P}\right) \leq Q(f) \leq \lambda .
$$


Thus,

$$
\int_{P} g d x=\int_{\{x \in Q: g(x)>\lambda\}} g(x) d x \leq c \lambda\left|\left\{x \in Q: M_{\mathfrak{D}(Q)} g(x)>\lambda\right\}\right|=c \lambda|P|,
$$

where we have used the facts that $M_{\mathfrak{D}(Q)} g(x)=Q(f) \leq \lambda$, when $x \in Q \backslash P$, and $M_{\mathfrak{D}(Q)} g(x)=P(f)>\lambda$, when $x \in P$. This proves $P(f) \leq c \lambda$.

Lemma 4.5. Suppose that the basis $\mathfrak{B}$ has dyadic substructure with the Stein property. Let $B \in \mathfrak{B}$.

(1) If the weight $w$ is in $A_{p, \mathfrak{D}(B)}, 1<p<\infty$, then there exists a sequence of weights $\left\{w_{k}\right\} \in A_{p, \mathfrak{D}(B)} \cap L^{\infty}(B)$ such that $\lim _{k \rightarrow \infty} w_{k}=w$ a.e.

(2) If the weight $w$ is in $\mathrm{RH}_{p, \mathfrak{D}(B)}, 1<p<\infty$, then there exists a sequence of weights $\left\{w_{k}\right\} \in \mathrm{RH}_{p, \mathfrak{D}(B)} \cap L^{\infty}(B)$ such that $\lim _{k \rightarrow \infty} w_{k}=w$ a.e.

Proof. Fix a $w \in A_{p, \mathfrak{B}}$. We perform the Calderón-Zygmund decomposition of $w$ at level $k, k \gg 1$, with respect to $\mathfrak{D}(B)$. Namely, let $\left\{Q_{k, j}\right\}_{j} \subset \mathfrak{D}(B)$ be the collection of dyadic bases in $\mathfrak{D}(B)$ which are maximal among the $Q \in \mathfrak{D}(B)$ that satisfy $Q(w)>k$. The maximality of $Q_{k, j}$ yields that the sets $Q_{k, j}$ are pairwise disjoint for varying $j$ and, by Lemma 4.4 , that $Q_{k, j}(w) \leq c k$. We now let

$$
w_{k}=w \mathbf{1}_{E_{k}}+\sum_{j} Q_{k, j}(w) \mathbf{1}_{Q_{k, j}},
$$

where $E_{k}=B \backslash \bigcup_{j} Q_{k, j}$. Then $w_{k}$ is in $L^{\infty}(B)$, because the basis $\mathfrak{D}(B)$ is a density basis, and $w_{k}$ converges to $w$ as $k \rightarrow \infty$, because

$$
\sum_{j}\left|Q_{k, j}\right| \leq \frac{w(B)}{k}
$$

To verify $w_{k} \in A_{p, \mathfrak{B}}$, we need only observe that

$$
1 \leq Q_{k, j}(w) Q_{k, j}\left(w^{-1 /(p-1)}\right)^{p-1}
$$

and hence

$$
Q_{k, j}(w)^{-1 /(p-1)} \leq Q_{k, j}\left(w^{-1 /(p-1)}\right) .
$$

When $w \in \mathrm{RH}_{p, \mathfrak{B}}$, we need only also observe that

$$
Q_{k, j}(w)^{p} \leq Q_{k, j}\left(w^{p}\right) .
$$

A well known result in the theory of weighted norm inequalities due to Gehring (cf. [7]) states that if $w \in \mathrm{RH}_{p, \mathfrak{Q}}$, then there exists $\varepsilon>0$ such that $w \in \mathrm{RH}_{p+\varepsilon, \mathfrak{Q}}$. Here, the symbol $\mathfrak{Q}$ denotes the collection of all cubes in $\mathbf{R}^{n}$ with sides parallel to the coordinate axes. We now extend this lemma to the general basis $\mathfrak{B}$.

Theorem 4.6. Suppose that the basis $\mathfrak{B}$ has dyadic substructure with the Stein property. Then, if $w \in \mathrm{RH}_{p, \mathfrak{B}}$ for some $1<p<\infty$, there exists $\varepsilon>0$ such that $w \in \mathrm{RH}_{p+\varepsilon, \mathfrak{B}}$.

Proof. Fix a $w \in \mathrm{RH}_{p, \mathfrak{B}}$. Given $B \in \mathfrak{B}$, we shall prove that the inequality

$$
\left(\frac{1}{|B|} \int_{B} w(x)^{p(1+\delta)} d x\right)^{1 /(p(1+\delta))} \leq C B(w)
$$

holds for some $C>0$ and $\delta>0$ independent of $B$. By a standard limiting argument based upon Lemma 4.5, we may assume without loss of generality that $w$ is in $L^{\infty}(B)$. 
We now let $a=B\left(w^{p}\right)$. It follows from Fubini's theorem that

$$
\begin{aligned}
\frac{1}{|B|} \int_{B} w(x)^{p(1+\delta)} d x & =\frac{\delta}{|B|} \int_{0}^{\infty} w^{p}\left(\left\{x \in B: w(x)^{p}>\lambda\right\}\right) \lambda^{\delta-1} d \lambda \\
& \leq a^{1+\delta}+\frac{\delta}{|B|} \int_{a}^{\infty} w^{p}\left(\left\{x \in B: w(x)^{p}>\lambda\right\}\right) \lambda^{\delta-1} d \lambda .
\end{aligned}
$$

The Stein property yields

$$
\begin{aligned}
& \frac{\delta}{|B|} \int_{a}^{\infty} w^{p}\left(\left\{x \in B: w(x)^{p}>\lambda\right\}\right) \lambda^{\delta-1} d \lambda \\
& \leq c \frac{\delta}{|B|} \int_{a}^{\infty}\left|\left\{x \in B: M_{\mathfrak{D}(B)}\left[w^{p} \mathbf{1}_{B}\right](x)>\lambda\right\}\right| \lambda^{\delta} d \lambda \\
& \leq c \frac{\delta}{(1+\delta)|B|} \int_{B} M_{\mathfrak{D}(B)}\left[w^{p} \mathbf{1}_{B}\right](x)^{1+\delta} d x .
\end{aligned}
$$

We notice that

$$
M_{\mathfrak{D}(B)}\left[w^{p} \mathbf{1}_{B}\right](x) \leq[w]_{\mathrm{RH}_{p, \mathfrak{B}}} M_{\mathfrak{D}(B)}\left[w \mathbf{1}_{B}\right](x)^{p} .
$$

Thus,

$$
\begin{aligned}
& \frac{\delta}{|B|} \int_{a}^{\infty} w^{p}\left(\left\{x \in B: w(x)^{p}>\lambda\right\}\right) \lambda^{\delta-1} d \lambda \\
& \leq \frac{C \delta}{(1+\delta)|B|} \int_{B} M_{\mathfrak{D}(B)}\left[w \mathbf{1}_{B}\right](x)^{p(1+\delta)} d x \\
& \leq \frac{C \delta}{1+\delta}\left\|M_{\mathfrak{D}(B)}\right\|_{L^{p(1+\delta)}\left(\mathbf{R}^{n}\right)}^{p(1+\delta)} \frac{1}{|B|} \int_{B} w(x)^{p(1+\delta)} d x .
\end{aligned}
$$

By using an elementary fact

$$
\lim _{\delta \rightarrow+0}\left\|M_{\mathfrak{D}(B)}\right\|_{L^{p(1+\delta)}\left(\mathbf{R}^{n}\right)}^{p(1+\delta)}=\left(p^{\prime}\right)^{p},
$$

we can select $\delta>0$ so that

$$
\frac{C \delta}{1+\delta}\left\|M_{\mathfrak{D}(B)}\right\|_{L^{p(1+\delta)}\left(\mathbf{R}^{n}\right)}^{p(1+\delta)}=\frac{1}{2}
$$

Hence, notice that the integral is finite, since $w$ is in $L^{\infty}(B)$ and $B$ is a bounded set,

$$
\frac{1}{|B|} \int_{B} w(x)^{p(1+\delta)} d x \leq 2 a^{1+\delta} \leq 2(C B(w))^{p(1+\delta)}
$$

which is our desired inequality.

The next theorem (Theorem 4.8) states that $A_{\infty, \mathfrak{B}} \subset \mathrm{RH}_{1, \mathfrak{B}}$. To prove the theorem, we need the following lemma, which is well-known, but, for the reader's convenience, we will quote the proof from the lecture notes of Hytönen "Weighted norm inequalities", since now we are not able to get this from his website.

Lemma 4.7. Let $\mathfrak{D}$ be a countable collection of measurable subsets of $\mathbf{R}^{n}$ with the property that

$$
\forall Q, R \in \mathfrak{D}: Q \cap R \in\{Q, R, \emptyset\} .
$$

Let $\mu$ be a positive measure on $\mathbf{R}^{n}$ and $f$ be a locally $\mu$-integrable function on $\mathbf{R}^{n}$. Define the maximal operator $M_{\mathfrak{D}, \mu}^{0}$ by

$$
M_{\mathfrak{D}, \mu}^{0} f(x)=\sup _{Q \in \mathfrak{D}} \mathbf{1}_{Q}(x) \exp \left(\frac{1}{\mu(Q)} \int_{Q} \log |f| d \mu\right) .
$$


Then, for all $p \in(0, \infty]$,

$$
\left\|M_{\mathfrak{D}, \mu}^{0} f\right\|_{L^{p}(\mu)} \leq e^{1 / p}\|f\|_{L^{p}(\mu)} .
$$

Proof. Define the maximal operator $M_{\mathfrak{D}, \mu}$ by

$$
M_{\mathfrak{D}, \mu} f(x)=\sup _{Q \in \mathfrak{D}} \mathbf{1}_{Q}(x) \frac{1}{\mu(Q)} \int_{Q}|f| d \mu .
$$

By Jensen's inequality and the basic properties of the logarithm, we have

$$
M_{\mathfrak{D}, \mu}^{0} f \leq M_{\mathfrak{D}, \mu} f, \quad\left(M_{\mathfrak{D}, \mu}^{0} f\right)^{p}=\left(M_{\mathfrak{D}, \mu}^{0}\left[|f|^{p / q}\right]\right)^{q} \leq\left(M_{\mathfrak{D}, \mu}\left[|f|^{p / q}\right]\right)^{q}, \quad q \in(0, \infty) .
$$

By the $L^{q}(\mu)$ boundedness of $M_{\mathfrak{D}, \mu}$ for $q>1$, we have

$$
\int_{\mathbf{R}^{n}}\left(M_{\mathfrak{D}, \mu}^{0} f\right)^{p} d \mu \leq \int_{\mathbf{R}^{n}}\left(M_{\mathfrak{D}, \mu}\left[|f|^{p / q}\right]\right)^{q} d \mu \leq\left(q^{\prime}\right)^{q} \int_{\mathbf{R}^{n}}\left(|f|^{p / q}\right)^{q} d \mu=\left(q^{\prime}\right)^{q} \int_{\mathbf{R}^{n}}|f|^{p} d \mu .
$$

As $q \rightarrow \infty$, we have $\left(q^{\prime}\right)^{q} \rightarrow e$, and hence

$$
\left\|M_{\mathfrak{D}, \mu}^{0} f\right\|_{L^{p}(\mu)}^{p} \leq e\|f\|_{L^{p}(\mu)}^{p} \quad \text { for } \quad p \in(0, \infty) .
$$

It is trivial for the case $p=\infty$.

Theorem 4.8. Suppose that the basis $\mathfrak{B}$ has a dyadic substructure with the Stein property. Then, if $w \in A_{\infty, \mathfrak{B}}$, there exists $\varepsilon>0$ such that $w \in \mathrm{RH}_{1+\varepsilon, \mathfrak{B}}$.

Proof. Fix a $w \in A_{\infty, \mathfrak{B}}$, that is, fix $w \in A_{p, \mathfrak{B}}$ for some large $p$. Given $B \in \mathfrak{B}$, we shall prove that the inequality

$$
\left(\frac{1}{|B|} \int_{B} w(x)^{1+\delta} d x\right)^{1 /(1+\delta)} \leq C B(w)
$$

holds for some $C>0$ and $\delta>0$ independent of $B$. In the same manner as above we may assume that $w$ is in $L^{\infty}(B)$.

We now let $a=B(w)$. It follows from Fubini's theorem that

$$
\begin{aligned}
\frac{1}{|B|} \int_{B} w(x)^{1+\delta} d x & =\frac{\delta}{|B|} \int_{0}^{\infty} w(\{x \in B: w(x)>\lambda\}) \lambda^{\delta-1} d \lambda \\
& \leq a^{1+\delta}+\frac{\delta}{|B|} \int_{a}^{\infty} w(\{x \in B: w(x)>\lambda\}) \lambda^{\delta-1} d \lambda .
\end{aligned}
$$

By the Stein property

$$
\begin{aligned}
& \frac{\delta}{|B|} \int_{a}^{\infty} w(\{x \in B: w(x)>\lambda\}) \lambda^{\delta-1} d \lambda \\
& \leq c \frac{\delta}{|B|} \int_{a}^{\infty}\left|\left\{x \in B: M_{\mathfrak{D}(B)}\left[w \mathbf{1}_{B}\right](x)>\lambda\right\}\right| \lambda^{\delta} d \lambda \\
& \leq c \frac{\delta}{(1+\delta)|B|} \int_{B} M_{\mathfrak{D}(B)}\left[w \mathbf{1}_{B}\right](x)^{1+\delta} d x .
\end{aligned}
$$

We notice that,

$$
M_{\mathfrak{D}(B)}\left[w \mathbf{1}_{B}\right](x) \leq[w]_{A_{p, \mathfrak{B}}} M_{\mathfrak{D}(B), d x}^{0}\left[w \mathbf{1}_{B}\right](x) .
$$

Thus, by Lemma 4.7,

$$
\begin{aligned}
& \frac{\delta}{|B|} \int_{a}^{\infty} w(\{x \in B: w(x)>\lambda\}) \lambda^{\delta-1} d \lambda \\
& \leq C \frac{\delta}{(1+\delta)|B|} \int_{B} M_{\mathfrak{D}(B), d x}^{0}\left[w \mathbf{1}_{B}\right](x)^{1+\delta} d x \leq \frac{C e \delta}{1+\delta} \frac{1}{|B|} \int_{B} w(x)^{1+\delta} d x .
\end{aligned}
$$


We select $\delta>0$ so that

Hence,

$$
\frac{C e \delta}{1+\delta}=\frac{1}{2}
$$

which is our desired inequality.

$$
\frac{1}{|B|} \int_{B} w(x)^{1+\delta} d x \leq 2 a^{1+\delta}=2 B(w)^{1+\delta}
$$

To verify the converse relation $\mathrm{RH}_{1, \mathfrak{B}} \subset A_{\infty, \mathfrak{B}}$, we must assume that $w$ satisfies the following assumption.

Assumption 4.9. Let $w$ be a weight. Suppose that $\mathfrak{B}$ has a dyadic substructure. There exists a uniform constant $c$ for varying $B \in \mathfrak{B}$ such that

$$
\int_{\{x \in B: f(x)>\lambda\}} f(x) w(x) d x \leq c \lambda w\left(\left\{x \in B: M_{\mathfrak{D}(B), w}\left[f \mathbf{1}_{B}\right](x)>\lambda\right\}\right)
$$

holds for any non-negative function $f \in L_{\text {loc }}^{1}(w d x), B \in \mathfrak{B}$ and $\lambda>w(B)^{-1} \int_{B} f w d x$.

Theorem 4.10. Suppose that $w$ satisfies Assumption 4.9. Then, if $w \in \mathrm{RH}_{s, \mathfrak{B}}$ for some $1<s<\infty$, there exists $p>1$ such that $w \in A_{p, \mathfrak{B}}$.

Proof. If $w \in \mathrm{RH}_{s, \mathfrak{B}}$, then for any $B \in \mathfrak{B}$

$$
B\left(w^{s}\right)^{1 / s} \leq[w]_{\mathrm{RH}_{s, \mathfrak{B}}} B(w) .
$$

This implies that

$$
\left(\frac{1}{w(B)} \int_{B} w(x)^{-1} w(x) d x\right)\left(\frac{1}{w(B)} \int_{B}\left(w(x)^{-1}\right)^{1-s} w(x) d x\right)^{s^{\prime}-1} \leq[w]_{\mathrm{RH}}^{s_{s, \mathfrak{B}}^{\prime}},
$$

which means that

$$
w^{-1} \in A_{s^{\prime}, \mathfrak{B}}^{w},
$$

where the class $A_{s^{\prime}, \mathfrak{B}}^{w}$ is defined by the Muckenhoupt class replacing the Lebesgue measure $d x$ by $w d x$. Thanks to Assumption 4.9 and Lemma 4.7, the same manipulation as in the proof of Theorem 4.8 gives us that the inequality

$$
\left(\frac{1}{w(B)} \int_{B}\left(w(x)^{-1}\right)^{1+\varepsilon} w(x) d x\right)^{1 /(1+\varepsilon)} \leq C \frac{|B|}{w(B)}
$$

holds for some $C>0$ and $\varepsilon>0$ independent of $B$. A calculation shows that

$$
\left(\frac{w(B)}{|B|}\right)^{\varepsilon /(1+\varepsilon)}\left(\frac{1}{|B|} \int_{B} w(x)^{-\varepsilon} d x\right)^{1 /(1+\varepsilon)} \leq C
$$

and that

$$
B(w) B\left(w^{-\varepsilon}\right)^{1 / \varepsilon} \leq C^{(1+\varepsilon) / \varepsilon}
$$

which means $w \in A_{p, \mathfrak{B}}$ with $1 /(p-1)=\varepsilon$.

In the remainder of this section we discuss an equivalent way of defining the reverse Hölder class $\mathrm{RH}_{1, \mathfrak{B}}$ (Theorem 4.12) following the papers [9] and [15].

Definition 4.11. A function $\Phi:[0, \infty) \rightarrow[0, \infty]$ is said to be a Young function if it is left-continuous, convex and increasing, and if $\Phi(0)=0$ and $\Phi(t) \rightarrow \infty$ as $t \rightarrow \infty$. Given a Young function $\Phi$, define the mean Luxemburg norm of $f$ on a finite set $B \subset \mathbf{R}^{n}$ by

$$
\|f\|_{\Phi, B}=\inf \left\{\lambda>0: \frac{1}{|B|} \int_{B} \Phi\left(\frac{|f(x)|}{\lambda}\right) d x \leq 1\right\} .
$$


If $\Phi(t)=t \log (e+t)$, we write the mean Luxemburg norm as $\|f\|_{L \log L, B}$.

Theorem 4.12. Suppose that the basis $\mathfrak{B}$ has a dyadic substructure with the Stein property. Then $w$ is in $\mathrm{RH}_{1, \mathfrak{B}}$ if and only if

$$
[w]_{\mathrm{RH}_{1, \mathfrak{B}}}=\sup _{B \in \mathfrak{B}} \frac{\|w\|_{L \log L, B}}{B(w)}<\infty .
$$

Before proving Theorem 4.12, we show two equivalent quantities to the quantity $[w]_{1, \mathfrak{B}}$.

Proposition 4.13. The following two quantities are equivalent to the quantity $[w]_{1, \mathfrak{B}}$ :

$$
[w]_{\mathrm{RH}_{1, \mathfrak{B}}}^{\prime}=\sup _{B \in \mathfrak{B}} \frac{1}{w(B)} \int_{B} w(x) \log \left(e+\frac{w(x)}{B(w)}\right) d x
$$

and

$$
[w]_{\mathrm{RH}_{1, \mathfrak{B}}^{\prime \prime}}^{\prime \prime} \sup _{B \in \mathfrak{B}} \frac{1}{w(B)} \int_{B} M_{\mathfrak{D}(B)} w(x) d x .
$$

The quantity $[w]_{\mathrm{RH}_{1, \mathfrak{B}}}^{\prime \prime}$ is known as Fujii-Wilson constant; see [9].

Proof of Proposition 4.13. If $[w]_{\mathrm{RH}}^{\prime}{ }_{1, \mathfrak{B}}=C_{2}<\infty$, then we have

$$
\frac{1}{|B|} \int_{B} \frac{w(x)}{B(w)} \log \left(e+\frac{w(x)}{B(w)}\right) d x=\frac{1}{w(B)} \int_{B} w(x) \log \left(e+\frac{w(x)}{B(w)}\right) d x<C_{2} .
$$

We notice that, for any Young function $\Phi, \Phi(\theta t) \leq \theta \Phi(t)$ holds for all $0<\theta<1$.

This fact and $C_{2}>1$ imply

$$
\frac{1}{|B|} \int_{B} \frac{w(x)}{C_{2} B(w)} \log \left(e+\frac{w(x)}{C_{2} B(w)}\right) d x \leq 1
$$

and, by the definition of the mean Luxemburg norm,

$$
\|w\|_{L \log L, B} \leq C_{2} B(w)
$$

which means that $[w]_{\mathrm{RH}_{1, \mathfrak{B}}} \leq C_{2}$.

If $[w]_{\mathrm{RH}_{1, \mathfrak{B}}}=C_{1}<\infty$, then we have

$$
\|w\|_{L \log L, B} \leq C_{1} B(w) .
$$

By the definition of the mean Luxemburg norm, we see that

$$
\frac{1}{|B|} \int_{B} \frac{w(x)}{C_{1} B(w)} \log \left(e+\frac{w(x)}{C_{1} B(w)}\right) d x \leq 1 .
$$

Noticing that $C_{1}>1$ and

$$
\frac{1}{C_{1}}\left(e+\frac{w(x)}{B(w)}\right) \leq\left(e+\frac{w(x)}{C_{1} B(w)}\right)
$$

we have

$$
1 \geq \frac{1}{w B)} \int_{B} \frac{w(x)}{C_{1}} \log \left(e+\frac{w(x)}{B(w)}\right) d x-\frac{\log C_{1}}{C_{1}}
$$

and

$$
\frac{1}{w B)} \int_{B} w(x) \log \left(e+\frac{w(x)}{B(w)}\right) d x \leq 2 C_{1},
$$

which means that $[w]_{\mathrm{RH}_{1, \mathfrak{B}}}^{\prime} \leq 2 C_{1}$. 

lemma.

The equivalence of the second and third quantities holds from the following

Lemma 4.14. Suppose that the basis $\mathfrak{B}$ has a dyadic substructure with the Stein property. Then, for any $B \in \mathfrak{B}$ and any measurable function $w$, we have

$$
\begin{aligned}
\frac{1}{1+c} \int_{B} w(x) \log \left(e+\frac{w(x)}{B(w)}\right) d x & \leq \int_{B} M_{\mathfrak{D}(B)}\left[w \mathbf{1}_{B}\right](x) d x \\
& \leq 10 \int_{B} w(x) \log \left(e+\frac{w(x)}{B(w)}\right) d x
\end{aligned}
$$

Proof. Fix a $B \in \mathfrak{B}$. By homogeneity, we may assume that $B(w)=1$. It follows from Fubini's theorem that

$$
\int_{B} w \log (e+w) d x=\int_{0}^{\infty} w(\{x \in B: w(x)>\lambda\}) \frac{d \lambda}{e+\lambda}=\int_{0}^{1} \cdots+\int_{1}^{\infty} \cdots=I+I I .
$$

There holds

$$
I \leq w(B) \leq \int_{B} M_{\mathfrak{D}(B)}\left[w \mathbf{1}_{B}\right] d x
$$

because $\mathfrak{D}(B)$ is a density basis, and by the Stein property

$$
\begin{aligned}
I I & =\int_{1}^{\infty} w(\{x \in B: w(x)>\lambda\}) \frac{d \lambda}{e+\lambda} \\
& \leq c \int_{1}^{\infty}\left|\left\{x \in B: M_{\mathfrak{D}(B)}\left[w \mathbf{1}_{B}\right](x)>\lambda\right\}\right| \frac{\lambda}{e+\lambda} d \lambda \\
& \leq c \int_{0}^{\infty}\left|\left\{x \in B: M_{\mathfrak{D}(B)}\left[w \mathbf{1}_{B}\right](x)>\lambda\right\}\right| d \lambda=c \int_{B} M_{\mathfrak{D}(B)}\left[w \mathbf{1}_{B}\right](x) d x .
\end{aligned}
$$

This gives the first inequality in (4.6). For the second inequality, we invoke the elementary inequality

$$
\left|\left\{x \in B: M_{\mathfrak{D}(B)}\left[w \mathbf{1}_{B}\right](x)>\lambda\right\}\right| \leq \frac{2}{\lambda} w(\{x \in B: w(x)>\lambda / 2\}), \quad \lambda>0 .
$$

This implies

$$
\begin{aligned}
\int_{2}^{\infty}\left|\left\{x \in B: M_{\mathfrak{D}(B)}\left[w \mathbf{1}_{B}\right](x)>\lambda\right\}\right| d \lambda & \leq 2 \int_{2}^{\infty} w(\{x \in B: w(x)>\lambda / 2\}) \frac{d \lambda}{\lambda} \\
& =2 \int_{1}^{\infty} w(\{x \in B: w(x)>\lambda\}) \frac{d \lambda}{\lambda} \\
& \leq 8 \int_{1}^{\infty} w(\{x \in B: w(x)>\lambda\}) \frac{d \lambda}{e+\lambda} \\
& \leq 8 \int_{B} w \log (e+w) d x .
\end{aligned}
$$

Noticing

$$
\int_{0}^{2}\left|\left\{x \in B: M_{\mathfrak{D}(B)}\left[w \mathbf{1}_{B}\right](x)>\lambda\right\}\right| d \lambda \leq 2|B|=2 w(B) \leq 2 \int_{B} w \log (e+w) d x,
$$

we obtain the second inequality in (4.6).

To prove Theorem 4.12, we need one more lemma. 
Lemma 4.15. Let $[w]_{\mathrm{RH}_{1, \mathfrak{B}}^{\prime}}=C_{2}<\infty$ and $B \in \mathfrak{B}$. Then for any measurable subset $S \subset B$ and $\lambda>0$, we have

$$
\frac{|S|}{|B|}<e^{-\lambda} \quad \text { implies } \quad \frac{w(S)}{w(B)} \leq \frac{2 C_{2}}{\lambda}+e^{-\lambda / 2} .
$$

Proof. Let

$$
E_{\lambda}=\left\{x \in B: w(x)>e^{\lambda} B(w)\right\}
$$

Then

$$
w\left(E_{\lambda}\right) \leq \frac{1}{\lambda} \int_{B} w(x) \log \left(e+\frac{w(x)}{B(w)}\right) d x \leq \frac{C_{2}}{\lambda} w(B)
$$

by (4.4). Therefore,

$$
\begin{aligned}
w(S) & =w\left(S \cap E_{\lambda / 2}\right)+w\left(S \backslash E_{\lambda / 2}\right) \leq \frac{2 C_{2}}{\lambda} w(B)+e^{\lambda / 2} B(w)|S| \\
& \leq \frac{2 C_{2}}{\lambda} w(B)+e^{\lambda / 2} e^{-\lambda} w(B) \quad \text { by the hypothesis in }(4.8) \\
& =\frac{2 C_{2}}{\lambda} w(B)+e^{-\lambda / 2} w(B) .
\end{aligned}
$$

This proves (4.8).

Remark 4.16. If $\lambda$ is sufficiently large, then we obtain $\alpha=e^{-\lambda}<1$ and $\beta=$ $\frac{2 C_{2}}{\lambda}+e^{-\lambda / 2}<1$. Thus, by $(4.8)$

$$
\frac{|S|}{|B|}<\alpha \quad \text { implies } \quad \frac{w(S)}{w(B)} \leq \beta .
$$

Proof of Theorem 4.12. Fix a $w \in \mathrm{RH}_{p, \mathfrak{B}}$ for some $1<p<\infty$. The fact that $t \log (e+t) \leq(1+e) t^{p}, t>1$, implies that

$$
[w]_{\mathrm{RH}_{1, \mathfrak{B}}} \leq(1+e)[w]_{\mathrm{RH}_{p, \mathfrak{B}}}<\infty .
$$

We shall prove the converse. We follow the interesting argument in $[15, \mathrm{p} .18$ proof of Theorem 5.4]. Let $[w]_{\mathrm{RH}_{1, \mathfrak{B}}}^{\prime}=C_{2}<\infty$. We have to prove that, given $B \in \mathfrak{B}$, the inequality

$$
\left(\frac{1}{|B|} \int_{B} w(x)^{1+\delta} d x\right)^{1 /(1+\delta)} \leq C B(w)
$$

holds for some $C>0$ and $\delta>0$ independent of $B$. Observe that by homogeneity we can assume that $B(w)=1$. In the same manner as in the proof of Lemma 4.5 based upon (4.5), we may assume without loss of generality that $w$ is in $L^{\infty}(B)$.

Set $a>1$ so that $\frac{c}{a}=\alpha$, where $c$ is the constant appearing in the definition of the Stein property and $\alpha$ is the constant in Remark 4.16. It follows that

$$
\begin{aligned}
\int_{B} w(x)^{1+\delta} d x & \leq \int_{B} M_{\mathfrak{D}(B)}\left[w \mathbf{1}_{B}\right](x)^{\delta} w(x) d x \\
& =\delta \int_{0}^{\infty} w\left(\left\{x \in B: M_{\mathfrak{D}(B)}\left[w \mathbf{1}_{B}\right](x)>\lambda\right\}\right) \lambda^{\delta-1} d \lambda \\
& \leq w(B)+\delta \sum_{k=0}^{\infty} \int_{c^{k}}^{c^{k+1}} w\left(\left\{x \in B: M_{\mathfrak{D}(B)}\left[w \mathbf{1}_{B}\right](x)>\lambda\right\}\right) \lambda^{\delta-1} d \lambda
\end{aligned}
$$




$$
\begin{aligned}
& \leq|B|+\delta c^{\delta} \sum_{k=0}^{\infty} \int_{a^{k}}^{a^{k+1}} w\left(\left\{x \in B: M_{\mathfrak{D}(B)}\left[w \mathbf{1}_{B}\right](x)>a^{k}\right\}\right) a^{k \lambda} \frac{d \lambda}{\lambda} \\
& =|B|+\delta a^{\delta} \log a \sum_{k=0}^{\infty} a^{k \lambda} w\left(\Omega_{k}\right),
\end{aligned}
$$

where

$$
\Omega_{k}=\left\{x \in B: M_{\mathfrak{D}(B)}\left[w \mathbf{1}_{B}\right](x)>a^{k}\right\} .
$$

Noticing $a^{k}>1=B(w)$, we can apply the Calderón-Zygmund decomposition of $w$ at level $a^{k}$ to obtain a family of maximal non-overlapping dyadic bases $\left\{Q_{k, j}\right\}_{j} \subset \mathfrak{D}(B)$ for which $\Omega_{k}=\bigcup_{j} Q_{k, j}$ and

$$
a^{k}<Q_{k, j}(w) \leq c a^{k},
$$

where we have used Lemma 4.4. Now,

$$
\sum_{k=0}^{\infty} a^{k \delta} w\left(\Omega_{k}\right)=\sum_{k, j} a^{k \delta} w\left(Q_{k, j}\right) \leq \sum_{k, j} Q_{k, j}(w)^{\delta} w\left(Q_{k, j}\right) .
$$

Let $E_{k, j}=Q_{k, j} \backslash \Omega_{k+1}$ and $F_{k, j}=Q_{k, j} \cap \Omega_{k+1}$. Of course, we have $\left|Q_{k, j}\right|=\left|E_{k, j}\right|+\left|F_{k, j}\right|$. There holds by (4.10) that

$$
\begin{aligned}
\left|F_{k, j}\right| & =\sum_{Q_{k+1, i} \subset Q_{k, j}}\left|Q_{k+1, i}\right| \leq \frac{1}{a^{k+1}} \sum_{Q_{k+1, i} \subset Q_{k, j}} w\left(Q_{k+1, i}\right) \\
& \leq \frac{1}{a^{k+1}} w\left(Q_{k, j}\right) \leq \frac{c a^{k}}{a^{k+1}}\left|Q_{k, j}\right|=\frac{c}{a}\left|Q_{k, j}\right|=\alpha\left|Q_{k, j}\right| .
\end{aligned}
$$

This implies by (4.9)

and hence

$$
\frac{w\left(F_{k, j}\right)}{w\left(Q_{k, j}\right)} \leq \beta
$$

$$
(1-\beta) w\left(Q_{k, j}\right) \leq w\left(E_{k, j}\right)
$$

We also notice that the sets $E_{k, j}$ are pairwise disjoint. Thus,

$$
\begin{aligned}
\sum_{k, j} Q_{k, j}(w)^{\delta} w\left(Q_{k, j}\right) & \leq \frac{1}{1-\beta} \sum_{k, j} Q_{k, j}(w)^{\delta} w\left(E_{k, j}\right) \\
& \leq \frac{1}{1-\beta} \sum_{k, j} \int_{E_{k, j}} M_{\mathfrak{D}(B)}\left[w \mathbf{1}_{B}\right](x)^{\delta} w(x) d x \\
& =\frac{1}{1-\beta} \int_{B} M_{\mathfrak{D}(B)}\left[w \mathbf{1}_{B}\right](x)^{\delta} w(x) d x
\end{aligned}
$$

Choosing $\delta$ so that

$$
\frac{\delta a^{\delta} \log a}{1-\beta}=\frac{1}{2}
$$

we obtain

$$
\int_{B} w(x)^{1+\delta} d x \leq 2|B|
$$

which is our desired inequality. 


\section{Volume formula}

In this section we extend the nice formula (1.2) to higher dimensions. Throughout this section we always assume that the weight $w$ is a radial weight: $w(x)=w_{0}(|x|)$, $x \in \mathbf{R}^{n}$, for some non-negative function $w_{0}$ on the half interval $[0, \infty)$. We suppose further that $w_{0}$ satisfies the supremum condition (1.1). When $w_{0}$ satisfies (1.1), it is not difficult to deduce that $w_{0}$ has the doubling condition:

$$
\int_{r_{1}}^{r_{2}} w_{0}(r) d r \leq C \int_{r_{1}^{\prime}}^{r_{2}^{\prime}} w_{0}(r) d r
$$

for all $0 \leq r_{1} \leq r_{1}^{\prime} \leq r_{2}^{\prime} \leq r_{2}<\infty$ with $r_{2}-r_{1}=2\left(r_{2}^{\prime}-r_{1}^{\prime}\right)$. For an $A \subset \mathbf{R}^{n}$ we set

$$
r_{1}(A)=\inf _{x \in A}|x|, \quad r_{2}(A)=\sup _{x \in A}|x| \quad \text { and } \quad \operatorname{rad}(A)=r_{2}(A)-r_{1}(A) .
$$

Let $\mathfrak{R}$ be the family of all rectangles in $\mathbf{R}^{n}, n \geq 2$, with sides parallel to the coordinate axes.

Lemma 5.1. Let $R \in \mathfrak{R}$ be any rectangle. Then

$$
\frac{w(R)}{|R|} \approx \frac{1}{\operatorname{rad}(R)} \int_{r_{1}(R)}^{r_{2}(R)} w_{0}(r) d r .
$$

Proof. Passing to polar coordinates, we observe that

$$
w(R)=\int_{r_{1}(R)}^{r_{2}(R)} \sigma\left(S_{r} \cap R\right) w_{0}(r) d r
$$

where $\mathcal{S}_{r}$ is sphere of radius $r$ and centered at the origin and $\sigma$ is its surface measure. Since $w_{0}$ satisfies the supremum condition (1.1), we get

$$
w(R) \leq \sup _{r_{1}(R)<r<r_{2}(R)} w_{0}(r) \int_{r_{1}(R)}^{r_{2}(R)} \sigma\left(R \cap \mathcal{S}_{r}\right) d r \leq C \frac{1}{\operatorname{rad}(R)} \int_{r_{1}(R)}^{r_{2}(R)} w_{0}(r) d r \cdot|R| .
$$

Thus, we shall prove the converse:

$$
w(R) \geq C \frac{1}{\operatorname{rad}(R)} \int_{r_{1}(R)}^{r_{2}(R)} w_{0}(r) d r \cdot|R| .
$$

Because of the rotation invariance and the symmetry of the problem, we may assume that the rectangle $R$ is of the form

$$
R=\prod_{i=1}^{n}\left(a_{i}, b_{i}\right), \quad 0<a_{i}<b_{i}<b_{1}<\infty .
$$

For $j=2, \ldots, n$, set $R_{j}=\left(a_{1}, b_{1}\right) \times\left(a_{j}, b_{j}\right)$. We now observe that

$$
\begin{aligned}
\operatorname{rad}(R) & =\sqrt{b_{1}^{2}+b_{2}^{2}+\cdots+b_{n}^{2}}-\sqrt{a_{1}^{2}+a_{2}^{2}+\cdots+a_{n}^{2}} \\
& =\frac{\left(b_{1}^{2}+b_{2}^{2}+\cdots+b_{n}^{2}\right)-\left(a_{1}^{2}+a_{2}^{2}+\cdots+a_{n}^{2}\right)}{\sqrt{b_{1}^{2}+b_{2}^{2}+\cdots+b_{n}^{2}}+\sqrt{a_{1}^{2}+a_{2}^{2}+\cdots+a_{n}^{2}}} \\
& \leq \sum_{j=2}^{n} \frac{\left(b_{1}^{2}+b_{j}^{2}\right)-\left(a_{1}^{2}+a_{j}^{2}\right)}{\sqrt{b_{1}^{2}+b_{j}^{2}}+\sqrt{a_{1}^{2}+a_{j}^{2}}} \\
& =\sum_{j=2}^{n}\left(\sqrt{b_{1}^{2}+b_{j}^{2}}-\sqrt{a_{1}^{2}+a_{j}^{2}}\right)=\sum_{j=2}^{n} \operatorname{rad}\left(R_{j}\right) .
\end{aligned}
$$


By this observation, we may further assume that

$$
\operatorname{rad}(R) \leq(n-1) \operatorname{rad}\left(R_{2}\right) .
$$

We now verify that

$$
\operatorname{rad}\left(R_{2}\right) \leq 2 \sqrt{n} \operatorname{rad}(R)
$$

Indeed,

$$
\begin{aligned}
\operatorname{rad}(R) & =\sqrt{b_{1}^{2}+b_{2}^{2}+\cdots+b_{n}^{2}}-\sqrt{a_{1}^{2}+a_{2}^{2}+\cdots+a_{n}^{2}} \\
& \geq \sqrt{\left(b_{1}^{2}+b_{2}^{2}\right)+\left(b_{3}^{2}+\cdots+b_{n}^{2}\right)}-\sqrt{\left(a_{1}^{2}+a_{2}^{2}\right)+\left(b_{3}^{2}+\cdots+b_{n}^{2}\right)} \\
& =\frac{\left(b_{1}^{2}+b_{2}^{2}\right)-\left(a_{1}^{2}+a_{2}^{2}\right)}{\sqrt{\left(b_{1}^{2}+b_{2}^{2}\right)+\left(b_{3}^{2}+\cdots+b_{n}^{2}\right)}+\sqrt{\left(a_{1}^{2}+a_{2}^{2}\right)+\left(b_{3}^{2}+\cdots+b_{n}^{2}\right)}} \\
& \geq \frac{\left(b_{1}^{2}+b_{2}^{2}\right)-\left(a_{1}^{2}+a_{2}^{2}\right)}{2 \sqrt{n} b_{1}} \geq \frac{1}{2 \sqrt{n}} \frac{\left(b_{1}^{2}+b_{2}^{2}\right)-\left(a_{1}^{2}+a_{2}^{2}\right)}{\sqrt{b_{1}^{2}+b_{2}^{2}}+\sqrt{a_{1}^{2}+a_{2}^{2}}} \\
& =\frac{1}{2 \sqrt{n}}\left(\sqrt{b_{1}^{2}+b_{2}^{2}}-\sqrt{a_{1}^{2}+a_{2}^{2}}\right)=\frac{\operatorname{rad}\left(R_{2}\right)}{2 \sqrt{n}} .
\end{aligned}
$$

Let $\bar{R}=\prod_{i=3}^{n}\left(a_{i}, b_{i}\right)$. It follows by Fubini's theorem that

$$
w(R)=\int_{\bar{R}}\left(\int_{R_{2}} w_{0}\left(\left(x_{1}^{2}+x_{2}^{2}+x_{3}^{2}+\cdots+x_{n}^{2}\right)^{1 / 2}\right) d x_{1} d x_{2}\right) d x_{3} \cdots d x_{n} .
$$

Fix $\bar{x} \in \bar{R}$ and let $h=|\bar{x}|$. We shall estimate

$$
I \equiv \int_{R_{2}} w_{0}\left(\left(x_{1}^{2}+x_{2}^{2}+h^{2}\right)^{1 / 2}\right) d x_{1} d x_{2} .
$$

In [18], it is shown that there exists a set $A \subset R_{2}$ such that

$$
\operatorname{rad}\left(R_{2}\right) \leq 8 \operatorname{rad}(A)
$$

and

$$
\operatorname{rad}(A) \inf _{r_{1}(A)<r<r_{2}(A)} \operatorname{arc}\left(A \cap \mathcal{C}_{r}\right) \geq \frac{\left|R_{2}\right|}{32},
$$

where $\mathcal{C}_{r}$ is the circle of radius $r$ and centered at the origin and $\operatorname{arc}\left(A \cap \mathcal{C}_{r}\right)$ is the arc length of the $\operatorname{arc} A \cap \mathcal{C}_{r}$. Moreover, it is also shown that

$$
r_{2}(A)=\max \left(\sqrt{b_{1}^{2}+a_{2}^{2}}, \sqrt{a_{1}^{2}+b_{2}^{2}}\right) .
$$

The inequality (5.5) yields

$$
\begin{aligned}
I & =\int_{r_{1}\left(R_{2}\right)}^{r_{2}\left(R_{2}\right)} w_{0}\left(\left(r^{2}+h^{2}\right)^{1 / 2}\right) \operatorname{arc}\left(R_{2} \cap \mathcal{C}_{r}\right) d r \\
& \geq \int_{r_{1}(A)}^{r_{2}(A)} w_{0}\left(\left(r^{2}+h^{2}\right)^{1 / 2}\right) \operatorname{arc}\left(A \cap \mathcal{C}_{r}\right) d r \\
& \geq \operatorname{rad}(A) \inf _{r_{1}(A)<r<r_{2}(A)} \operatorname{arc}\left(A \cap \mathcal{C}_{r}\right) \cdot \frac{1}{\operatorname{rad}(A)} \int_{r_{1}(A)}^{r_{2}(A)} w_{0}\left(\left(r^{2}+h^{2}\right)^{1 / 2}\right) d r \\
& \geq \frac{1}{\operatorname{rad}(A)} \int_{r_{1}(A)}^{r_{2}(A)} w_{0}\left(\left(r^{2}+h^{2}\right)^{1 / 2}\right) d r \cdot \frac{\left|R_{2}\right|}{32} .
\end{aligned}
$$


There holds by (5.3)

$$
\operatorname{rad}(A) \leq \operatorname{rad}\left(R_{2}\right) \leq 2 \sqrt{n} \operatorname{rad}(R)
$$

Using the change of variables $t=\left(r^{2}+h^{2}\right)^{1 / 2}$, we have

$$
\begin{aligned}
\int_{r_{1}(A)}^{r_{2}(A)} w_{0}\left(\left(r^{2}+h^{2}\right)^{1 / 2}\right) d r & =\int_{\left(r_{1}(A)^{2}+h^{2}\right)^{1 / 2}}^{\left(r_{2}(A)^{2}+h^{2}\right)^{1 / 2}} w_{0}(t) \frac{t}{\sqrt{t^{2}-h^{2}}} d t \\
& \geq \int_{\left(r_{1}(A)^{2}+h^{2}\right)^{1 / 2}}^{\left(r_{2}(A)^{2}+h^{2}\right)^{1 / 2}} w_{0}(t) d t .
\end{aligned}
$$

It follows that

$$
\operatorname{rad}(A) \leq 2 \sqrt{n}\left(\sqrt{r_{2}(A)^{2}+h^{2}}-\sqrt{r_{1}(A)^{2}+h^{2}}\right)
$$

Indeed,

$$
\begin{aligned}
& \sqrt{r_{2}(A)^{2}+h^{2}}-\sqrt{r_{1}(A)^{2}+h^{2}}=\frac{r_{2}(A)^{2}-r_{1}(A)^{2}}{\sqrt{r_{2}(A)^{2}+h^{2}}+\sqrt{r_{1}(A)^{2}+h^{2}}} \\
& \geq \frac{r_{2}(A)^{2}-r_{1}(A)^{2}}{2 \sqrt{n} b_{1}} \geq \frac{r_{2}(A)^{2}-r_{1}(A)^{2}}{2 \sqrt{n} \sqrt{b_{1}^{2}+a_{2}^{2}}} \\
& \geq \frac{1}{2 \sqrt{n}} \frac{r_{2}(A)^{2}-r_{1}(A)^{2}}{r_{2}(A)+r_{1}(A)}=\frac{r_{2}(A)-r_{1}(A)}{2 \sqrt{n}}=\frac{\operatorname{rad}(A)}{2 \sqrt{n}} \quad \text { by }(5.6),
\end{aligned}
$$

where we have used (5.6). By the inequalities (5.2), (5.4) and (5.8), we see that there exists a open interval $(a, b) \subset\left(\left(r_{1}(A)^{2}+h^{2}\right)^{1 / 2},\left(r_{2}(A)^{2}+h^{2}\right)^{1 / 2}\right) \subset\left(r_{1}(R), r_{2}(R)\right)$ such that

$$
\operatorname{rad}(R)=16(n-1) \sqrt{n}(b-a) .
$$

This yields, thanks to the doubling condition 5.1,

$$
\int_{\left(r_{1}(A)^{2}+h^{2}\right)^{1 / 2}}^{\left(r_{2}(A)^{2}+h^{2}\right)^{1 / 2}} w_{0}(r) d r \geq \int_{a}^{b} w_{0}(r) d r \geq C \int_{r_{1}(R)}^{r_{2}(R)} w_{0}(r) d r .
$$

Thus, by (5.7) and (5.9),

$$
I \geq C \frac{1}{\operatorname{rad}(R)} \int_{r_{1}(R)}^{r_{2}(R)} w_{0}(r) d r \cdot\left|R_{2}\right| .
$$

Integrating both sides of this inequality over $\bar{R}$, we obtain

$$
w(R) \geq C \frac{1}{\operatorname{rad}(R)} \int_{r_{1}(R)}^{r_{2}(R)} w_{0}(r) d r \cdot|R|,
$$

which completes the proof.

Fix $N \gg 1$. Let $\mathfrak{K}_{N}$ be the family of all tubes in $\mathbf{R}^{n}, n \geq 2$, with eccentricity $N$ (the ratio of the length of long-sides and short-sides is equal to $N$ ). Lemma 5.1 yields the following.

Theorem 5.2. Suppose that $w_{0}:[0, \infty) \rightarrow[0, \infty)$ satisfies $(1.1)$. Let $w(x)=$ $w_{0}(|x|), x \in \mathbf{R}^{n}$, be a radial weight. Then the weight $w$ belongs to the reverse Hölder class $\mathrm{RH}_{\infty, \Re}$ and the reverse Hölder class $\mathrm{RH}_{\infty, \mathfrak{K}_{N}}$. 
Proof. Lemma 5.1 and (1.1) imply, for any $R \in \mathfrak{R}$,

$R(w)=\frac{w(R)}{|R|} \geq C \frac{1}{\operatorname{rad}(R)} \int_{r_{1}(R)}^{r_{2}(R)} w_{0}(r) d r \geq C \sup _{r_{1}(R)<r<r_{2}(R)} w_{0}(r)=C \operatorname{essips}_{x \in R} w(x)$,

which means that $w$ belongs to $\mathrm{RH}_{\infty, \Re}$. For the case $T \in \mathfrak{K}_{N}$, let $L$ be the axis of the tube $T$ and let $\Pi$ be the plane which contains the origin and $L$. Rotating $\Pi$ to the plane $\left(x_{1}, x_{2}\right)$ and applying another rotation in the plane $\left(x_{1}, x_{2}\right)$, we may assume that $T$ is of the form

$$
T=\left(a_{1}, b_{1}\right) \times\left(a_{2}, b_{2}\right) \times(-c / 2, c / 2)^{n-2},
$$

where $0<a_{1}<b_{1}, 0<a_{2}<b_{2}, b_{1}-a_{1}=N c, b_{2}-a_{2}=c$ and $c>0$. This observation, Lemma 5.1 and (1.1) yield that $w$ belongs to $\mathrm{RH}_{\infty, \mathfrak{K}_{N}}$.

It is well-known that the strong maximal operator $M_{\mathfrak{R}}$ is bounded on $L^{p}\left(\mathbf{R}^{n}\right)$ for $p>1$; see for example [6, p. 452]. It is also known that the Kakeya maximal operator $M_{\mathfrak{K}_{N}}$ satisfies for $1<p \leq(n+2) / 2$ (see [22, Theorem 1] and, for the log-factors, [21, Theorem 1]. The result of [21] is based on the argument in [19].)

$$
\left\|M_{\mathfrak{K}_{N}}\right\|_{L^{p}\left(\mathbf{R}^{n}\right)} \leq C N^{n / p-1}(\log N)^{\alpha_{n}} .
$$

These facts, Corollary 2.7 and Theorem 5.2 yield the following.

Proposition 5.3. Suppose that $w_{0}:[0, \infty) \rightarrow[0, \infty)$ satisfies $(1.1)$. Let $w(x)=$ $w_{0}(|x|), x \in \mathbf{R}^{n}$, be a radial weight. Then,

(a) for $1<p \leq \infty$,

$$
\left\|M_{\mathfrak{R}, w} f\right\|_{L^{p}(w)} \leq C\|f\|_{L^{p}(w)} .
$$

(b) for $1<p \leq(n+2) / 2$,

$$
\left\|M_{\mathfrak{K}_{N}, w} f\right\|_{L^{p}\left(\mathbf{R}^{n}, w\right)} \leq C N^{n / p-1}(\log N)^{\alpha_{n}}\|f\|_{L^{p}\left(\mathbf{R}^{n}, w\right)},
$$

where the constants $C$ and $\alpha_{n}$ are independent of $N$.

\section{References}

[1] Alfonseca, A., F. Soria, and A. Vargas: A remark on maximal operators along directions in $\mathbf{R}^{2}$. - Math. Res. Lett. 10:1, 2003, 41-49.

[2] Alfonseca, A., F. Soria, and A. VArgas: An almost-orthogonality principle in $L^{2}$ for directional maximal functions. - In: Harmonic analysis at Mount Holyoke (South Hadley, MA, 2001), Contemp. Math. 320, Amer. Math. Soc., Providence, RI, 2003, 1-7.

[3] CórdobA, A.: The Kakeya maximal function and the spherical summation multiplier. - Amer. J. Math. 99:1, 1977, 1-22.

[4] Cruz-Uribe, D., and C. J. Neugebauer: The structure of the reverse Hölder classes. - Trans. Amer. Math. Soc. 347:8, 1995, 2941-2960.

[5] Duonndikoetxea, J., F. J. Martín-Reyes, and S. Ombrosi: On the $A_{\infty}$ conditions for general bases. - Math. Z. 282, 2016, 955-972.

[6] Garcia-Cuerva, J., and J. L. Rubio de Francia: Weighted norm inequalities and related topics. - Math. Stud. 116, North-Holland, 1985).

[7] Gehring, F. W.: The $L^{p}$ integrability of partial derivatives of a quasiconformal mapping. Acta Math. 130, 1973, 265-277.

[8] Hagelstein, P., T. Luque, and I. Parissis: Tauberian conditions, Muckenhoupt weights, and differentiation properties of weighted bases. - Trans. Amer. Math. Soc. 367:11, 2015, 79998032.

[9] Hytönen, T., and C. PÉREz: Sharp weighted bounds involving $A_{\infty}$. - Anal. PDE 6:4, 2013, $777-818$. 
[10] JAWERTH, B.: Weighted inequalities for maximal operators: linearization, localization and factorization. - Amer. J. Math. 108:2, 1986, 361-414.

[11] Lerner, A. K.: An elementary approach to several results on the Hardy-Littlewood maximal operator. - Proc. Amer. Math. Soc. 136:8, 2008, 2829-2833.

[12] Lerner, A. K., and S. Ombrosi: A boundedness criterion for general maximal operators. Publ. Mat. 54:1, 2010, 53-71.

[13] PÉrez, C.: A remark on weighted inequalities for general maximal operators. Proc. Amer. Math. Soc. 119:4, 1993, 1121-1126.

[14] PÉREz, C.: Weighted norm inequalities for general maximal operators. - In: Conference on Mathematical Analysis (El Escorial, 1989), Publ. Mat. 35:1, 1991, 169-186.

[15] PÉrez, C.: The theory of $A_{p}$ weights: a modern introduction. - Preprint.

[16] Strömberg, J. O.: Maximal functions associated to rectangles with uniformly distributed directions. - Ann. of Math. (2) 107:2, 1978, 399-402.

[17] Saito, H., and Y. Sawano: A note on the Kakeya maximal operator and radial weights on the plane. - Tôhoku Math. J. (to appear).

[18] Saito, H., and H. TANAKa: Directional maximal operators and radial weights on the plane. - Bull. Austral. Math. Soc. 89:2, 2014, 397-414.

[19] Sogge, C.: Concerning Nikodym-type sets in 3-dimensional curved spaces. - J. Amer. Math. Soc. 12:1, 1999, 1-31.

[20] Stein, E. M.: Note on the class $L \log L$. - Studia Math. 32, 1969, 305-310.

[21] Tanaka, H.: The Fefferman-Stein type inequality for the Kakeya maximal operator in Wolff's range. - Proc. Amer. Math. Soc. 133:3, 2005, 763-772.

[22] Wolff, T.: - An improved bound for Kakeya type maximal functions. - Rev. Mat. Iberoam. 11:3, 1995, 651-674.

Received 2 November 2015 • Accepted 2 September 2016 